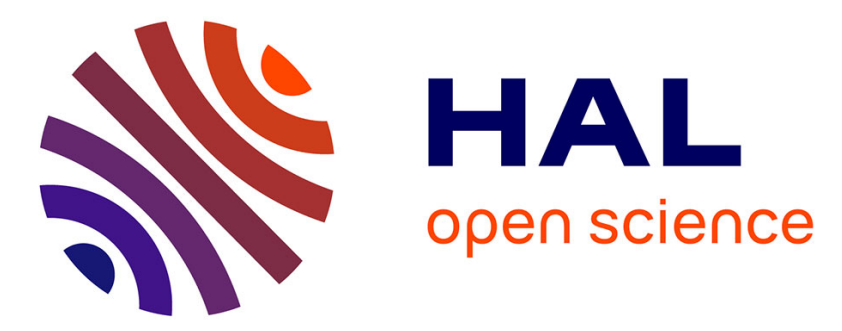

\title{
Eulerian and Hamiltonian dicycles in directed hypergraphs
}

Julio Araujo, Jean-Claude Bermond, Guillaume Ducoffe

\section{To cite this version:}

Julio Araujo, Jean-Claude Bermond, Guillaume Ducoffe. Eulerian and Hamiltonian dicycles in directed hypergraphs. Discrete Mathematics, Algorithms and Applications, 2014, 06, pp.1450012. 10.1142/S1793830914500128 . hal-01104634v2

\section{HAL Id: hal-01104634 \\ https://hal.science/hal-01104634v2}

Submitted on 6 Feb 2015

HAL is a multi-disciplinary open access archive for the deposit and dissemination of scientific research documents, whether they are published or not. The documents may come from teaching and research institutions in France or abroad, or from public or private research centers.
L'archive ouverte pluridisciplinaire HAL, est destinée au dépôt et à la diffusion de documents scientifiques de niveau recherche, publiés ou non, émanant des établissements d'enseignement et de recherche français ou étrangers, des laboratoires publics ou privés. 


\title{
Eulerian and Hamiltonian Dicycles in Directed Hypergraphs ${ }^{* \dagger}$
}

\author{
JULIO ARAUJO ${ }^{\ddagger}$ JEAN-CLAUDE BERMOND \\ COATI Project, INRIA and I3S (CNRS/UNS). \\ 2004, Route des Lucioles - B.P. 93 - F-06902 Sophia Antipolis \\ Cedex, France. \\ julio.araujo@inria.fr, jean-claude.bermond@inria.fr \\ GUILLAUME DUCOFFE \\ University of Nice-Sophia Antipolis, France. \\ gducoffe@polytech.unice.fr
}

\begin{abstract}
In this article, we generalize the concepts of Eulerian and Hamiltonian digraphs to directed hypergraphs. A dihypergraph $H$ is a pair $(\mathcal{V}(H), \mathcal{E}(H))$, where $\mathcal{V}(H)$ is a non-empty set of elements, called vertices, and $\mathcal{E}(H)$ is a collection of ordered pairs of subsets of $\mathcal{V}(H)$, called $h y$ perarcs. It is Eulerian (resp. Hamiltonian) if there is a dicycle containing each hyperarc (resp. each vertex) exactly once. We first present some properties of Eulerian and Hamiltonian dihypergraphs. For example, we show that deciding whether a dihypergraph is Eulerian is an NP-complete problem. We also study when iterated line dihypergraphs are Eulerian and Hamiltonian. Finally, we study when the generalized de Bruijn dihypergraphs are Eulerian and Hamiltonian. In particular, we determine when they contain a complete Berge dicycle, i.e. an Eulerian and Hamiltonian dicycle.
\end{abstract}

\section{Introduction}

Eulerian and Hamiltonian dicycles are well-known concepts in Graph Theory. An Eulerian dicycle in a digraph $D$ is a dicycle $C$ such that each arc of $D$

\footnotetext{
*This research was supported by ANR Agape and Gratel.

${ }^{\dagger}$ Preprint submitted to the journal Discrete Mathematics, Algorithms and Applications.

${ }^{\ddagger}$ Supported by CNPq/Brazil under contract PDE 202049/2012-4.
} 
appears exactly once in $C$. Similarly, a Hamiltonian dicycle is a dicycle $C$ such that each vertex of $D$ appears exactly once in $C$ (see $[1,2]$ ).

We generalize these concepts to directed hypergraphs, called shortly dihypergraphs. Informally, the difference between an usual digraph $D$ and a dihypergraph $H$ is that (hyper)arcs in $H$ may have multiple heads and multiple tails. Formally, a dihypergraph $H$ is a pair $(\mathcal{V}(H), \mathcal{E}(H))$, where $\mathcal{V}(H)$ is a non-empty set of elements, called vertices, and $\mathcal{E}(H)$ is a collection of ordered pairs of subsets of $\mathcal{V}(H)$, called hyperarcs. It is Eulerian (resp. Hamiltonian) if there is a dicycle containing each hyperarc (resp. each vertex) exactly once.

Eulerian and Hamiltonian (undirected) hypergraphs have already been defined and studied in a similar way [3, 4]. In fact, if Hamiltonian hypergraphs have received some attention (see $[5,6,7]$ ), Eulerian hypergraphs seem to have been considered in their full generality only recently in [4]. A particular case of Eulerian cycles in 3-uniform hypergraphs (called triangulated irregular networks) has been considered in $[8,9,10]$ motivated by applications in geographic systems or in computer graphics. However, to our best knowledge, Hamiltonian and Eulerian dihypergraphs have not been considered.

Note that there are other definitions of Hamiltonian hypergraphs in the literature. For example, an undirected hypergraph $H$ is called Hamiltonian if there exists a Hamiltonian- $l$ cycle $C$ in $H$, that is a cycle $C$ where any two consecutive (hyper)edges intersect themselves in exactly $l$ vertices and every vertex of $H$ belongs to exactly one of those intersections [11, 6, 7]. Such a notion can also be generalized to dihypergraphs. However, we choose the general definition as otherwise there would be no more a clear connexion between the Eulerian and the Hamiltonian dihypergraphs (with our definition the dual of an Eulerian dihypergraph is Hamiltonian). Furthermore, we are mainly interested in Hamiltonian line dihypergraphs, whose definition is given later, and, in this case, both of these definitions of a Hamiltonian dihypergraph are equivalent.

It is well-known that a strongly connected digraph is Eulerian if, and only if, every vertex has equal in-degree and out-degree. Therefore, deciding whether a digraph is Eulerian can be done in polynomial time; but deciding whether it is Hamiltonian is an NP-complete problem.

In the first part of the article, we show that for dihypergraphs the situation is different from that of digraphs. For example, deciding whether a dihypergraph is Eulerian is an NP-complete problem. We show nonetheless that some results about the Eulerian digraphs can be generalized, in the case where the studied dihypergraphs are uniform and regular. As example, we prove that if $H$ is a weakly-connected, $d$-regular, $s$-uniform dihypergraph, then, for every $k \geq 1$, $L^{k}(H)$ is Eulerian and Hamiltonian. In the second part, we study the Eulerian and Hamiltonian properties of special families of regular uniform dihypergraphs, the generalized de Bruijn and Kautz dihypergraphs [12].

The so called de Bruijn digraphs were introduced to show the existence of de Bruijn sequences, that is circular sequences of $d^{D}$ elements, such that any subsequence of length $D$ appears exactly once. To prove the existence of such sequences, it was proved that de Bruijn digraphs are both Eulerian and Hamiltonian. These digraphs have been rediscovered many times and their 
properties have been well studied (see, for example, the survey [13]) in particular for the design of interconnection networks. Various generalizations of de Bruijn digraphs have been introduced, like the generalized de Bruijn digraphs (also named Reddy-Pradhan-Kuhl digraphs) presented in [14, 15]. These digraphs are based on arithmetical properties and they exist for any number of vertices. Other generalizations like Kautz digraphs, generalized Kautz digraphs (also called Imase and Itoh digraphs [14]) and consecutive digraphs [16] have been proposed in the literature.

One generalization concerns hypergraphs and dihypergraphs which are used in the design of optical bus networks [17]. In particular, de Bruijn and Kautz dihypergraphs and their generalizations, that were introduced in [12], have several properties that are beneficial in the design of large, dense, robust networks. They have been proposed as the underlying physical topologies for optical networks, as well as dense logical topologies for Logically Routed Networks (LRN) because of ease of routing, load balancing and congestion reduction, that are properties inherent in de Bruijn and Kautz networks. In 2009, J-J. Quisquater brought to our attention the web site (http://punetech.com/building-eka-theworlds-fastest-privately-funded-supercomputer/) where it is explained how these hypergraphs and the results of [18] were used for the design of the supercomputer EKA in 2007 (http://en.wikipedia.orwiki/EKA_(supercomputer)).

Connectivity properties of generalized de Bruijn dihypergraphs have been studied in [19, 20, 21], but, to our best knowledge, their Hamiltonian and Eulerian properties have not been studied.

More precisely, we first determine when generalized de Bruijn and Kautz dihypergraphs are Hamiltonian and Eulerian. Then, we study the case where their number of hyperarcs is equal to their number of vertices. In that case, we almost characterize when these dihypergraphs have a complete Berge dicycle, i.e. a dicycle both Hamiltonian and Eulerian; in particular, we have a complete characterization when the out-degree of each vertex is equal to the out-size of each hyperarc.

\section{Definitions and Notations}

\subsection{Dihypergraphs}

A directed hypergraph, or simply dihypergraph is a pair $(\mathcal{V}(H), \mathcal{E}(H))$ where $\mathcal{V}(H)$ is a non-empty set of elements, called vertices, and $\mathcal{E}(H)$ is a collection of ordered pairs of subsets of $\mathcal{V}(H)$, called hyperarcs. We denote by $n(H)$ (resp. $m(H))$ the number of vertices (resp. hyperarcs) of $H$. Whenever $H$ is clear in the context, we use shortly $n$ and $m$. We suppose, to avoid trivial cases, that $n>1$ and $m>1$ and that we have no isolated vertex.

Let $H$ be a dihypergraph and $E=\left(E^{-}, E^{+}\right)$be a hyperarc in $\mathcal{E}(H)$. Then, the vertex sets $E^{-}$and $E^{+}$are called the in-set and the out-set of the hyperarc $E$, respectively. The sets $E^{-}$and $E^{+}$do not need to be disjoint and they may be empty. The vertices of $E^{-}$are said to be incident to the hyperarc $E$ and the 
vertices of $E^{+}$are said to be incident from $E$.

If $E$ is a hyperarc in a dihypergraph $H$, then $\left|E^{-}\right|$is the in-size and $\left|E^{+}\right|$ is the out-size of $E$. The maximum in-size and the maximum out-size of $H$ are respectively:

$$
s^{-}(H)=\max _{E \in \mathcal{E}(H)}\left|E^{-}\right| \text {and } s^{+}(H)=\max _{E \in \mathcal{E}(H)}\left|E^{+}\right| .
$$

Note that a digraph is a dihypergraph $D=(\mathcal{V}(D), \mathcal{E}(D))$ with $s^{-}(D)=$ $s^{+}(D)=1$.

Let $v$ be a vertex in $H$. The in-degree of $v$ is the number of hyperarcs that contain $v$ in their out-set and it is denoted by $d_{H}^{-}(v)$. Similarly, the out-degree of vertex $v$ is the number of hyperarcs that contain $v$ in their in-set and it is denoted by $d_{H}^{+}(v)$.

The bipartite representation $R(H)$ of a dihypergraph $H$ is the bipartite digraph $R(H)=\left(\mathcal{V}_{1}(R) \cup \mathcal{V}_{2}(R), \mathcal{E}(R)\right)$ where $\mathcal{V}_{1}(R)=\mathcal{V}(H), \mathcal{V}_{2}(R)=\mathcal{E}(H)$ and $\mathcal{E}(R)=\left\{v_{i} E_{j} \mid v_{i} \in E_{j}^{-}\right\} \cup\left\{E_{j} v_{i} \mid v_{i} \in E_{j}^{+}\right\}$. This representation digraph is useful for drawing dihypergraphs. To make each figure more readable, we duplicate the vertices and we put in the left part the $\operatorname{arcs}$ from $\mathcal{V}_{1}$ to $\mathcal{V}_{2}$ and in the right part those from $\mathcal{V}_{2}$ to $\mathcal{V}_{1}$. Figure 1 gives the representation digraph of the de Bruijn dihypergraph $G B H(2,9,2,9)$ (see Section5), where vertex $i$ belongs to the in-set of the hyperarcs $E_{2 i}$ and $E_{2 i+1}$ and the hyperarc $E_{j}$ has as out-set the vertices $2 j$ and $2 j+1$ (all the numbers being taken modulo 9 ).

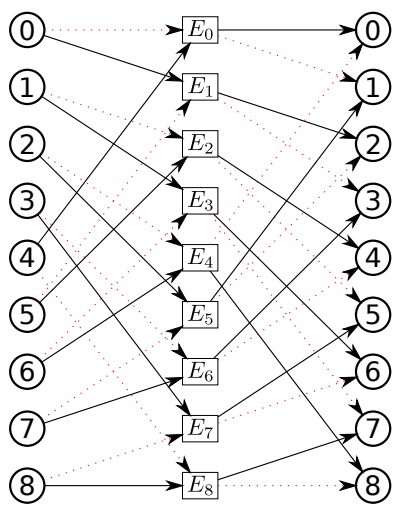

Figure 1: Bipartite representation of the De Bruijn dihypergraph $G B H(2,9,2,9)$ and a complete Berge dicycle represented by dotted arcs (vertices are drawn twice to better represent all the arcs).

Remark that when you inverse the respective roles of $\mathcal{V}_{1}(R)$ and $\mathcal{V}_{2}(R)$ in $R(H)$, you intuitively exchange the role of the vertices with the role of the hyperarcs in $H$. This is an informal notion of the dual dihypergraph $H^{*}$. Formally, the vertices of the dual dihypergraph $H^{*}$ are in bijection $\phi_{v}$ with the hyperarcs of $H$ and the hyperarcs of $H^{*}$ are in bijection $\phi_{E}$ with the vertices of 
$H$. Moreover, for every vertex $v \in \mathcal{V}(H)$ and every hyperarc $E \in \mathcal{E}(H)$, vertex $e=\phi_{v}(E) \in \mathcal{V}\left(H^{*}\right)$ is in $V^{-}$, where $V=\phi_{E}(v) \in \mathcal{E}\left(H^{*}\right)$, if, and only if, $v \in E^{+}$ and, similarly, $e$ is in $V^{+}$if, and only if, $v \in E^{-}$. It is important to notice that a hyperarc $V \in \mathcal{E}\left(H^{*}\right)$ may have an empty in-set (if $d_{H}^{-}(v)=0$ ) or an empty out-set (if $d_{H}^{+}(v)=0$ ).

The underlying multidigraph $U(H)$ of a dihypergraph $H$ has as vertex set $\mathcal{V}(U(H))=\mathcal{V}(H)$ and as arc set $\mathcal{E}(U(H))$ that is the multiset of all ordered pairs $(u, v)$ such that $u \in E^{-}$and $v \in E^{+}$, for every hyperarc $E \in \mathcal{E}(H)$. We emphasize that $U(H)$ does not need to be simple: the number of $\operatorname{arcs}$ from $u$ to $v$ in $U(H)$ is the number of hyperarcs $E=\left(E^{-}, E^{+}\right)$in $H$ such that $u \in E^{-}$ and $v \in E^{+}$. Observe that the underlying multidigraph of a given dihypergraph is unique. However, a given digraph $D$ can be the underlying digraph of many dihypergraphs $H$.

\subsection{Eulerian and Hamiltonian Dicycles in Dihypergraphs}

By a dipath in a dihypergraph $H$, we mean a sequence $P=v_{0}, E_{0}, \ldots, v_{p-1}, E_{p-1}, v_{p}$, such that, for all $i, j$, we have $v_{i} \in \mathcal{V}(H), E_{j} \in \mathcal{E}(H), v_{i} \in E_{i}^{-}$for every $0 \leq i \leq p-1$, and $v_{i} \in E_{i-1}^{+}$for every $1 \leq i \leq p$. We also say that $P$ is a dipath of length $p$. Moreover, the dipath $P$ is called a dicycle, or circuit, in $H$ if, and only if, we have $v_{0}=v_{p}$. Observe that each dicycle in a dihypergraph $H$ corresponds to a dicycle in its bipartite representation $R(H)$. Note that we allow repetitions of vertices or hyperarcs and some authors prefer to use the word tour in this case.

In the same way, we can extend the digraph-theoretic notions of Eulerian dicycles and Hamiltonian dicycles to dihypergraphs:

Definition 1. Let $H$ be a dihypergraph. We say that $H$ is Eulerian (resp. $H$ is Hamiltonian) if, and only if, there is a dicycle $C$ in $H$ such that every hyperarc of $H$ (resp. every vertex of $H$ ) appears in $C$ exactly once. We call $C$ an Eulerian dicycle (resp. a Hamiltonian dicycle).

Our generalization of an Eulerian dicycle to dihypergraphs is close to the extension of an Euler tour to the undirected hypergraphs introduced in [4].

Definition $2([4])$. Let $H_{u}$ be an undirected hypergraph. A tour is a sequence $T=v_{0}, E_{0}, v_{1}, \ldots$,

$v_{m-1}, E_{m-1}, v_{0}$ where, for all $i, v_{i} \neq v_{i+1}$ and $v_{i}$ and $v_{i+1}$ are in the hyperedge $E_{i}$ (indices are taken modulo $m$ ). $T$ is called an Euler tour when every hyperedge of $H_{u}$ appears exactly once in $T . H_{u}$ is an Eulerian hypergraph if there exists an Euler tour $\mathrm{T}$ in $H_{u}$.

Remark 1. An Eulerian dicycle in $H$ (resp. a Hamiltonian dicycle in $H$ ) is a dicycle in $R(H)$, such that each vertex of $\mathcal{V}_{2}(R)$ (resp. of $\mathcal{V}_{1}(R)$ ) appears exactly once. 
As a consequence, a necessary and sufficient condition for $R(H)$ to be Hamiltonian is that there is a dicycle $C$ in $H$, such that $C$ is simultaneously an Eulerian dicycle and a Hamiltonian dicycle in $H$. In reference to the undirected case [3], we call $C$ a complete Berge dicycle:

Definition 3. Let $H$ be a dihypergraph. A complete Berge dicycle in $H$ is a dicycle $C$ in $H$, such that $C$ is both an Eulerian dicycle and a Hamiltonian dicycle in $H$.

\section{General Results}

In the following sections, we focus on Eulerian dihypergraphs. Recall that we assume that the studied dihypergraphs have no isolated vertex, and that $n>1$ and $m>1$.

\subsection{Some conditions}

First, we recall a well-known characterization of Eulerian digraphs:

Theorem 1 ([22]). Let $D$ be a digraph. The following statements are equivalent:

1. $D$ is Eulerian;

2. $D$ is (strongly) connected and, for all vertex $v \in \mathcal{V}(D), d^{-}(v)=d^{+}(v)$;

3. $D$ is (strongly) connected and it has a dicycle decomposition (i.e. its arcs can be partitioned into arc-disjoint dicycles).

The digraph-theoretic notions of connectivity can be extended to dihypergraphs [19]. We say that $H$ is strongly (resp. weakly) connected if its underlying multidigraph $U(H)$ is strongly (resp. weakly) connected. $U(H)$ is weakly connected if its associated multigraph $G_{U(H)}$ (obtained by forgetting the orientation) is a connected multigraph (in Graph Theory this undirected graph is often called the underlying graph; we use here a different terminology as we already use the word underlying for the digraph associated to a dihypergraph). The digraph-theoretic notions of vertex-connectivity and arc-connectivity are also generalized by the dihypergraph-theoretic notions of vertex-connectivity and hyperarc-connectivity (see [19]). Unlike 1-arc connected digraphs, 1-hyperarc connected dihypergraphs are not always 1-vertex connected.

Remark that unlike an Eulerian digraph, an Eulerian dihypergraph does not need to be strongly connected. Indeed, let $H$ be an Eulerian dihypergraph. If

we add a new vertex $x$ in $H$, such that $x$ is incident to only one hyperarc $E$ of $H$ and $d^{-}(x)=0$, then the dihypergraph obtained is still Eulerian, but it is not strongly connected.

On the other hand, we have the following necessary condition:

Proposition 2. Let $H$ be a dihypergraph. If $H$ is Eulerian, then $H$ is weakly connected. 
Proof. Let $G_{U(H)}$ be the undirected associated multigraph to $U(H)$. We want to prove that $G_{U(H)}$ is connected. Note first that for all hyperarc $E \in \mathcal{E}(H)$, vertices in the subset $E^{-} \cup E^{+}$are in the same connected component in $G_{U(H)}$, by the definition of $U(H)$. Moreover, let $E, F$ be any pair of distinct hyperarcs of $H$. Since there is an Eulerian dicycle in $H$, therefore, there exist $u \in E^{+}$ and $v \in F^{-}$, such that there is a dipath in $H$ from $u$ to $v$. Since there is a dipath from $u$ to $v$ in $H$, therefore there is a dipath $P$ from $u$ to $v$ in $U(H)$ and so a path between $u$ and $v$ in $G_{U(H)}$. Therefore, the subsets $E^{-} \cup E^{+}$and $F^{-} \cup F^{+}$are in the same connected component in $G_{U(H)}$ too. Therefore, $G_{U(H)}$ is connected.

Recall that a hypergraph is $k$-uniform if all its hyperedges have the same cardinality $k$. It was proved in [4] that, if $H$ is an Eulerian $k$-uniform hypergraph, then $\left|\mathcal{V}_{\text {odd }}(H)\right| \leq(k-2) m(H)$, where $\mathcal{V}_{\text {odd }}(H)$ is the set of all the vertices in $H$ with an odd degree and $m(H)$ is the number of hyperedges in $H$. Using the same idea, we also prove a necessary condition for a dihypergraph $H$ to be Eulerian.

Theorem 3. Let $H$ be a dihypergraph. If $H$ is Eulerian then:

$$
\sum_{v \in \mathcal{V}(H)}\left|d^{+}(v)-d^{-}(v)\right| \leq \sum_{E \in \mathcal{E}(H)}\left(\left|E^{+}\right|+\left|E^{-}\right|-2\right) .
$$

Proof. Let $C=v_{0}, E_{0}, v_{1}, \ldots, v_{m-1}, E_{m-1}, v_{0}$ be an Eulerian dicycle in $H$. By definition, a given vertex may appear many times in $C$, but every hyperarc appears exactly once in the dicycle $C$. Let us find the maximum number of occurences of a given vertex $v$ in $C$. For all $i \neq j$ we may have $v_{i}=v_{j}$, but we are sure that $E_{i} \neq E_{j}$. So a vertex $v$ can appear at most $\min \left(d^{+}(v), d^{-}(v)\right)$ times in $C$ and, as a consequence, we have the following inequality:

$$
\sum_{v \in \mathcal{V}(H)} \min \left(d^{+}(v), d^{-}(v)\right) \geq m
$$

Moreover, we know that:

$$
\begin{aligned}
& \min \left(d^{+}(v), d^{-}(v)\right)=\frac{1}{2}\left(d^{+}(v)+d^{-}(v)-\left|d^{+}(v)-d^{-}(v)\right|\right), \\
& \sum_{v \in \mathcal{V}(H)} d^{+}(v)=\sum_{E \in \mathcal{E}(H)}\left|E^{-}\right| \text {and } \sum_{v \in \mathcal{V}(H)} d^{-}(v)=\sum_{E \in \mathcal{E}(H)}\left|E^{+}\right| .
\end{aligned}
$$

Therefore, the following inequalities hold:

$$
\begin{array}{r}
\sum_{v \in \mathcal{V}(H)}\left(d^{+}(v)+d^{-}(v)-\left|d^{+}(v)-d^{-}(v)\right|\right) \geq 2 m \\
\sum_{E \in \mathcal{E}(H)}\left|E^{-}\right|+\sum_{E \in \mathcal{E}(H)}\left|E^{+}\right|-\sum_{v \in \mathcal{V}(H)}\left|d^{+}(v)-d^{-}(v)\right| \geq \sum_{E \in \mathcal{E}(H)} 2 \\
\sum_{v \in \mathcal{V}(H)}\left|d^{+}(v)-d^{-}(v)\right| \leq \sum_{E \in \mathcal{E}(H)}\left[\left(\left|E^{+}\right|-1\right)+\left(\left|E^{-}\right|-1\right)\right]
\end{array}
$$


For a digraph $D$, Theorem 3 is

the Euler's condition presented in Theorem 1: for all $v \in \mathcal{V}(D), d^{+}(v)=$ $d^{-}(v)$.

Theorem 3 is not a sufficient condition for a strongly connected dihypergraph $H$ to be Eulerian: counter-examples are presented in Figure 2 and in Figure 3(b).

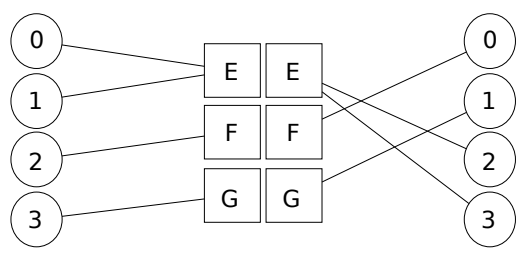

Figure 2: A regular dihypergraph that is not Eulerian.

Another necessary condition was proposed by N. Cohen (private communication), who transposed the search of an Eulerian dicycle into a PERFEct Matching problem (see $[3,2])$.

Let $H$ be a dihypergraph. If there is a hyperarc $E \in \mathcal{E}(H)$ whose in-set (resp. whose out-set) is empty, then $H$ cannot be Eulerian. Else, let $\varphi: \mathcal{E}(H) \rightarrow$ $\mathcal{V}(H) \times \mathcal{V}(H)$ be any function such that, for all $E$, we have $\varphi(E) \in E^{-} \times E^{+}$. By replacing each hyperarc $E$ by the arc $\varphi(E)$ we get a digraph, denoted by $D_{\varphi}[H]=(\mathcal{V}(H), \varphi(\mathcal{E}(H)))$. Observe that $D_{\varphi}[H]$ is a subdigraph of $U(H)$ and it can have loops or multiple arcs.

Remark 2. A dihypergraph $H$ is Eulerian if, and only if, there exists a function $\varphi$ such that $D_{\varphi}[H]$ is an Eulerian digraph.

By Theorem 1, a necessary and sufficient condition for a digraph $D$ to be Eulerian is that $D$ is connected and, for every vertex $v, d^{-}(v)=d^{+}(v)$. If $D$ satisfies this degree constraint for every vertex, but is not necessarily connected, we call $D$ a balanced digraph.

We will use the well-known Hall's Theorem to prove a necessary and sufficient condition for the digraph $D_{\varphi}[H]$ to be balanced, for some $\varphi$.

Theorem 4 (see $[3,2]$ ). Let $G=\left(\mathcal{V}_{1} \cup \mathcal{V}_{2}, \mathcal{E}\right)$ be a bipartite graph such that $\left|\mathcal{V}_{1}\right|=\left|\mathcal{V}_{2}\right|$. There is a perfect matching in $B$ if, and only if, for every subset $S \subset \mathcal{V}_{1},|\Gamma(S)| \geq|S|$, where $\Gamma(S)$ denotes the set of vertices adjacent to some vertex of $S$.

Definition 4. Let $X$ be a subset of $\mathcal{V}(H)$. We denote by $d_{H}^{+}(X)$ (shortly $d^{+}(X)$ ) the number of hyperarcs $E \in \mathcal{E}(H)$ such that $E^{-} \cap X \neq \emptyset$ and by $d_{s, H}^{-}(X)$ (shortly denoted $\left.d_{s}^{-}(X)\right)$ the number of hyperarcs $E$ such that $E^{+} \subseteq X$.

We are now able to prove the following result: 
Theorem 5. Let $H$ be a dihypergraph. There exists a function $\varphi$ such that $D_{\varphi}[H]$ is a balanced digraph if, and only if, for every subset $X \subseteq \mathcal{V}(H)$, we have $d_{s}^{-}(X) \leq d^{+}(X)$.

Proof. Let us assume there exists $\varphi$ such that $D_{\varphi}[H]$ is a balanced digraph. For every subset $X \subseteq \mathcal{V}(H)$, for every hyperarc $E$ such that $E^{+} \subseteq X$, we necessarily have $(u, v)=\varphi(E) \in \mathcal{V}(H) \times X$. Since $D_{\varphi}[H]$ is balanced, hence there must be some hyperarc $F$ such that $\varphi(F)$ has vertex $v$ as origin, that is $v \in F^{-}$. So $F^{-} \cap X$ is not empty. Furthermore, we can associate to two distinct hyperarcs $E$ two distinct hyperarcs $F$; therefore $d_{s}^{-}(X) \leq d^{+}(X)$.

Conversely, let us assume that for every subset $X \subseteq \mathcal{V}(H), d_{s}^{-}(X) \leq$ $d^{+}(X)$. Consider the bipartite graph $B P(H)=\left(\mathcal{V}_{1}(B P) \cup \mathcal{V}_{2}(B P), \mathcal{E}(B P)\right)$ with $\mathcal{V}_{1}(B P)=\left\{E_{j}^{+}: E_{j} \in \mathcal{E}(\mathcal{H})\right\}, \mathcal{V}_{2}(B P)=\left\{E_{j}^{-}: E_{j} \in \mathcal{E}(\mathcal{H})\right\}$ and $\mathcal{E}(B P)=\left\{E_{j}^{+} E_{j^{\prime}}^{-}: E_{j}^{+} \cap E_{j^{\prime}}^{-} \neq \emptyset\right\}$.

Let $S=\left\{E_{j_{1}}^{+}, E_{j_{2}}^{+}, \ldots, E_{j_{|S|}}^{+}\right\}$be a subset of $\mathcal{V}_{1}(B P)$ and $X=\bigcup_{k=1}^{|S|} E_{j_{k}}^{+}$. Observe that $|\Gamma(S)|=d^{+}(X)$. Since $d_{s}^{-}(X) \geq|S|$, we conclude that $|\Gamma(S)| \geq$ $|S|$. Moreover, $\mathcal{V}_{1}(B P)$ and $\mathcal{V}_{2}(B P)$ have the same cardinality. By Theorem 4 , there is a perfect matching $M$ in $B P(H)$. We now define a function $\varphi$ as follows: for all edge $E_{i}^{+} E_{j}^{-}$of $M$, one can choose any vertex $v \in E_{i}^{+} \cap E_{j}^{-}$as the tail of $\varphi\left(E_{i}\right)$ and the head of $\varphi\left(E_{j}\right)$. Thus, we get a subdigraph $D_{\varphi}[H]$ that is a balanced digraph.

Observe that we may define $d^{-}(X)$ and $d_{s}^{+}(X)$ in the same way as $d^{+}(X)$ and $d_{s}^{-}(X)$. Thus, another formulation of Theorem 5 is: there exists $\varphi$ such that $D_{\varphi}[H]$ is a balanced digraph if, and only if, for every subset $X \subseteq \mathcal{V}(H)$ $d_{s}^{+}(X) \leq d^{-}(X)$.

By Theorem 5, deciding whether there exists $\varphi$ such that $D_{\varphi}[H]$ is a balanced digraph can be done in polynomial time. However, deciding whether there exists $\varphi$ such that $D_{\varphi}[H]$ is strongly connected is an NP-complete problem [23].

\subsection{Duality and Complexity}

First, we show that the search of an Eulerian dicycle in $H$ is equivalent to the search of a Hamiltonian dicycle in its dual:

Proposition 6. A dihypergraph $H$ is Eulerian if, and only if, $H^{*}$ is Hamiltonian.

Proof. For each dicycle $C=v_{0}, E_{0}, v_{1}, E_{1}, \ldots, v_{p}, E_{p}, v_{0}$ of $H$ one can find a corresponding dicycle in $H^{*}$ namely $C^{*}=e_{0}, V_{1}, e_{1}, \ldots, e_{p}, V_{0}, e_{0}$ and vice-versa. Thus, $C$ is an Eulerian dicycle in $H$ (i.e. $C$ contains each hyperarc of $H$ exactly once) if, and only if, $C^{*}$ contains each vertex of $H^{*}$ exactly once, i.e. $C^{*}$ is a Hamiltonian dicycle of $H^{*}$.

As a direct consequence we can observe that, since $\left(H^{*}\right)^{*}=H, H$ is Hamiltonian if, and only if, $H^{*}$ is Eulerian. Moreover, since deciding whether a 
di(hyper)graph $H$ is Hamiltonian is an NP-complete problem [1], the following result is not surprising:

Theorem 7. Deciding whether a dihypergraph $H$ is Eulerian is NP-complete.

Proof. Let $C$ be a dipath. One can verify, in $O(|\mathcal{E}(H)|)$ operations, whether $C$ is an Eulerian dicycle in $H$. Consequently, the problem is in $N P$. Since the dual $H^{*}$ can be built in $O(|\mathcal{E}(H)|+|\mathcal{V}(H)|)$-time, we conclude the proof directly from Proposition 6 and the NP-completeness of deciding whether a digraph is Hamiltonian.

In order to check if a given dihypergraph is Hamiltonian, we will often use the following proposition:

Proposition 8. A dihypergraph $H$ is Hamiltonian if, and only if, its underlying multidigraph $U(H)$ is Hamiltonian.

Proof. By definition of $U(H)$, any dicycle in $H$ is a dicycle in $U(H)$ with the same vertices, and reciprocally.

\subsection{Line Dihypergraphs Properties}

The line dihypergraph $L(H)$ of a dihypergraph $H$ has as vertices the dipaths of length 1 in $H$ and as hyperarcs the dipaths of length 1 in $H^{*}$ :

$$
\begin{aligned}
& \mathcal{V}(L(H))=\bigcup_{E \in \mathcal{E}(H)}\left\{(u E v) \mid u \in E^{-}, v \in E^{+}\right\}, \\
& \mathcal{E}(L(H))=\bigcup_{v \in \mathcal{V}(H)}\left\{(E v F) \mid v \in E^{+} \cap F^{-}\right\} ;
\end{aligned}
$$

where the in-set and the out-set of hyperarc $(E v F)$ are $(E v F)^{-}=\{(u E v) \mid u \in$ $\left.E^{-}\right\}$and $(E v F)^{+}=\left\{(v F w) \mid w \in F^{+}\right\}$.

Particularly, when $D$ is a digraph, $L(D)$ is the line digraph of $D$ (see [1]).

The following results are used in the sequel:

Theorem 9 ([19]). Let $H$ be a dihypergraph. Then,

1. the digraphs $R(L(H))$ and $L^{2}(R(H))$ are isomorphic;

2. the digraphs $U(L(H))$ and $L(U(H))$ are isomorphic;

3. the digraphs $(L(H))^{*}$ and $L\left(H^{*}\right)$ are isomorphic.

Recall that:

Theorem 10 ([24]). For a given digraph $D$, the line digraph $L(D)$ is Hamiltonian if, and only if, $D$ is Eulerian. 
This property is useful for some special families of digraphs, e.g. Kautz and de Bruijn digraphs, that are stable by line digraph operation [24]. By using induction, one can prove that every digraph of the family is Hamiltonian. It was shown in [19] that de Bruijn and Kautz dihypergraphs are also stable by line dihypergraph operation. So, it is natural to wonder whether this property can be generalized to dihypergraphs. However, we only get a weak generalization.

Proposition 11. Let $H$ be a dihypergraph. Then, $L(H)$ is Hamiltonian if, and only if, $U(H)$ is Eulerian.

Proof. By Proposition 8, the dihypergraph $L(H)$ is Hamiltonian if, and only if, $U(L(H))$ is Hamiltonian. Moreover, $U(L(H))$ and $L(U(H))$ are isomorphic by Theorem 9. Finally, by Theorem $10 L(U(H))$ is Hamiltonian if, and only if, $U(H)$ is Eulerian.

We now show with two counter-examples that both implications of the corresponding version of Theorem 10 to dihypergraphs do not hold. There exist dihypergraphs which are Eulerian such that their line dihypergraph is not Hamiltonian and there also exist dihypergraphs that are not Eulerian such that their line dihypergraph is Hamiltonian.

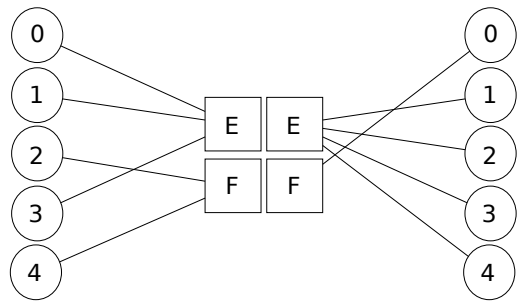

(a) $H_{1}$

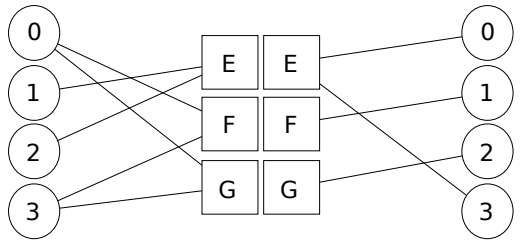

(b) $\mathrm{H}_{2}$

Figure 3: Counter-examples for extension of Theorem 10 to dihypergraphs.

Consider the dihypergraph $H_{1}=\left(\mathcal{V}\left(H_{1}\right), \mathcal{E}\left(H_{1}\right)\right)$ whose bipartite representation digraph is depicted in Figure 3(a). Observe that $0, E, 2, F, 0$ is an Eulerian dicycle in $H_{1}$. But $d_{U\left(H_{1}\right)}^{+}(1)=4$, that is different than $d_{U\left(H_{1}\right)}^{-}(1)=3$. As a consequence, $U\left(H_{1}\right)$ cannot be Eulerian, by Theorem 1 .

On the other hand, the dihypergraph $H_{2}=\left(\mathcal{V}\left(H_{2}\right), \mathcal{E}\left(H_{2}\right)\right)$, depicted in Figure 3(b), is not Eulerian, but $U(H)$ is Eulerian and so $L(H)$ is Hamiltonian. Remark that $H_{2}$ verifies the necessary condition of Theorem 3. Furthermore, $\mathrm{H}_{2}$ is strongly connected. One may observe that its underlying multidigraph $U\left(H_{2}\right)$ is Eulerian (it is even a 2-regular digraph). However, $H_{2}$ is not Eulerian because it does not verify the condition of Theorem 5 . Indeed, $d_{s}^{-}(\{1,2\})=2$, which is strictly greater than $d^{+}(\{1,2\})=1$.

We will show, in the next sections, that there are Eulerian dihypergraphs $H$, which are not digraphs, such that their $U(H)$ is Eulerian. 


\section{Case of $d$-regular, $s$-uniform Dihypergraphs}

Let $\left(s^{-}, s^{+}\right)$be a couple of positive integers. An $\left(s^{-}, s^{+}\right)$-uniform dihypergraph $H$ is a dihypergraph such that the in-size (resp. the out-size) of every hyperarc in $H$ equals $s^{-}$(resp. equals $s^{+}$). When $s^{-}=s^{+}=s$ we also say that $H$ is a $s$-uniform dihypergraph. Recall that digraphs are 1-uniform dihypergraphs.

Let $\left(d^{-}, d^{+}\right)$be a couple of positive integers. A $\left(d^{-}, d^{+}\right)$-regular dihypergraph $H$ is a dihypergraph such that the in-degree (resp. the out-degree) of every vertex in $H$ equals $d^{-}$(resp. $d^{+}$). When $d^{-}=d^{+}=d$, we also say that $H$ is a $d$-regular dihypergraph. Regular 1-uniform dihypergraphs are exactly regular digraphs. Remark that a dihypergraph $H$ is $(p, q)$-uniform if, and only if, its dual dihypergraph $H^{*}$ is $(p, q)$-regular, for any positive integers $p, q$.

When the studied dihypergraphs are uniform, Theorem 3 can be reformulated in a very similar way to [4]:

Corollary 1. Let $H$ be an Eulerian dihypergraph. If $H$ is $\left(s^{-}, s^{+}\right)$-uniform, then:

$$
\sum_{v \in \mathcal{V}(H)}\left|d^{+}(v)-d^{-}(v)\right| \leq\left(s^{+}+s^{-}-2\right) m
$$

Observe that even though $d$-regular dihypergraphs always verify the necessary condition of Theorem 3, they are not always Eulerian (see Figure 2).

We recall the following result about regular digraphs:

Theorem 12 ([1]). Deciding whether a 2-regular digraph $D$ is Hamiltonian is an NP-complete problem.

In [4], the authors use a similar result about 3-regular graphs, to prove that deciding whether a $k$-uniform hypergraph, $k \geq 3$, is Eulerian is an NP-complete problem. We do the same for uniform dihypergraphs. First, observe that if the dihypergraphs are 1-uniform, that is they are digraphs, we know that deciding whether a digraph is Eulerian can be done in polynomial time [24].

Theorem 13. Let $\left(s^{-}, s^{+}\right)$be a couple of positive integers. If $s^{-} \geq 2$ or $s^{+} \geq 2$, then deciding whether a $\left(s^{-}, s^{+}\right)$-uniform dihypergraph is Eulerian is an $N P$ complete problem.

Proof. By symmetry, we only need to prove the case when $s^{+} \geq 2$. Furthermore, by Theorem 7, we already know that the problem is in the NP-class. We now reduce the Hamiltonian problem in 2-regular digraphs to the Eulerian problem in $\left(s^{-}, s^{+}\right)$-uniform dihypergraphs.

The idea consists in associating in polynomial time to a 2-regular digraph $D$ a dihypergraph $H_{D}$, such that $H_{D}$ is Eulerian if, and only if, $D$ is Hamiltonian and then the result will follow by Theorem 12 .

Let $D=(\mathcal{V}(D), \mathcal{E}(D))$ be a 2-regular digraph. We define the dihypergraph $H_{D}$ with the following rules:

1. $\mathcal{V}\left(H_{D}\right)=\mathcal{V}(D) \cup\{A \times \mathcal{V}(D)\} \cup\{B \times \mathcal{V}(D)\}$, where $A$ and $B$ are two sets satisfying $|A|=s^{-}-1$ and $|B|=s^{+}-2$; 
2. to each vertex $v \in \mathcal{V}(D)$, we associate a hyperarc $E_{v} \in \mathcal{E}\left(H_{D}\right)$ such that $E_{v}^{-}=\{v\} \cup\{A \times\{v\}\}$ and $E_{v}^{+}=\left\{w_{v}, w_{v}^{\prime}\right\} \cup\{B \times\{v\}\}$, where $w_{v}$ and $w_{v}^{\prime}$ are the out-neighbors of $v$ in $D$.

By construction, $H_{D}$ is a $\left(s^{-}, s^{+}\right)$-uniform dihypergraph. Let us prove now that $D$ is Hamiltonian if, and only if, $H_{D}$ is Eulerian.

Suppose first that $D$ is Hamiltonian and let $C=v_{0}, v_{1}, \ldots, v_{n-1}, v_{0}$ be a Hamiltonian dicycle in $D$. From $C$, we build a dicycle $\mathcal{C}_{D}$ in $H_{D}, \mathcal{C}_{D}=$ $v_{0}, E_{v_{0}}, v_{1}, E_{v_{1}}, \ldots, v_{n-1}, E_{v_{n-1}}, v_{0}$, where $E_{v_{i}}$ is the hyperarc that is induced by $v_{i}$. By definition of a Hamiltonian dicycle, for all $v \in \mathcal{V}(D), v$ appears only once in $C$. Therefore, by construction of $H_{D}$, for every $E_{v} \in \mathcal{E}\left(H_{D}\right), E_{v}$ appears exactly once in $\mathcal{C}_{D}$. So, $\mathcal{C}_{D}$ is an Eulerian dicycle in $H_{D}$.

Now, suppose that $H_{D}$ is Eulerian. Remark that for every $E, F \in \mathcal{E}\left(H_{D}\right)$, by construction of $H_{D}$, we have $E^{+} \cap F^{-} \subset \mathcal{V}(D)$. Thus, let $\mathcal{C}_{D}=v_{0}, E_{0}, v_{1}, E_{1}, \ldots, v_{m-1}, E_{m-1}, v_{0}$ be an Eulerian dicycle in $H_{D}$. Because of the previous remark, we know that for every $i, v_{i} \in \mathcal{V}(D)$. However, a vertex $v \in \mathcal{V}(D)$ is incident to only one hyperarc in $H_{D}$. As a consequence, for all $i, E_{i}$ is the hyperarc that is associated to $v_{i}$ and so, each $v_{i}$ appears exactly once, and therefore, $C=v_{0}, v_{1}, \ldots, v_{m-1}, v_{0}$ is a Hamiltonian dicycle in $D$.

When $H$ is a digraph, we know that:

Theorem 14 ([24]). Let $D$ be a weakly-connected digraph. If $D$ is regular, then all its iterated line digraphs $L^{k}(D)$, for every $k \geq 1$, are Hamiltonian and Eulerian.

We now prove that, more generally:

Theorem 15. Let $H$ be a weakly-connected, d-regular, s-uniform dihypergraph. Then for every $k \geq 1, L^{k}(H)$ is Eulerian and Hamiltonian.

Proof. Since $H$ is $d$-regular and $s$-uniform, then $U(H)$ is a $d s$-regular multidigraph. As a consequence, for all $k \geq 0, L^{k}(U(H))$ is also $d s$-regular. By Theorem 9 , we have by induction on $k$ that, for all $k \geq 0, U\left(L^{k}(H)\right)$ is isomorphic to $L^{k}(U(H))$. So, for all $k \geq 0, U\left(L^{k}(H)\right)$ is Eulerian (because it is a regular multidigraph), that is equivalent, by Proposition 11, to $L\left(L^{k}(H)\right)=L^{k+1}(H)$ be Hamiltonian.

Moreover, $H^{*}$ is $s$-regular, $d$-uniform, and we claim that it is also a weakly connected dihypergraph. Indeed, $s \geq 1$ implies that there is no empty in-set and no empty out-set in $H^{*}$. So, the connectivity of $H$ implies the connectivity of $H^{*}$. Therefore, for every $k \geq 1 L^{k}\left(H^{*}\right)$ is also Hamiltonian. Again by Theorem 9 , we prove by induction on $k$ that, for all $k \geq 1,\left(L^{k}(H)\right)^{*}$ is isomorphic to $L^{k}\left(H^{*}\right)$. Therefore, by Proposition 6 , for every $k \geq 1 L^{k}(H)$ is Eulerian.

Remark 3. Theorem 15 holds when $H$ is $\left(d^{-}, d^{+}\right)$-regular, $H$ is $\left(s^{-}, s^{+}\right)$uniform, if we add the extra-condition: $d^{-} s^{-}=d^{+} s^{+}$. 
Recall that a complete Berge dicycle is an Eulerian and Hamiltonian dicycle and that if a dihypergraph has such a dicycle, then its bipartite representation digraph $R(H)$ is Hamiltonian. In the case $s=d$, we are able to prove a slightly more general result:

Proposition 16. Let $H$ be a d-regular, d-uniform dihypergraph that is weakly connected. There is a complete Berge dicycle in $L(H)$.

Proof. Because of the $d$-regularity, $d$-uniformity of $H$, its own bipartite representation digraph $R(H)$ is $d$-regular. Therefore, for every $i \geq 1, L^{i}(R(H))$ is Hamiltonian. By Theorem 9, we know that $L^{2}(R(H))$ and $R(L(H))$ are isomorphic. Therefore, $R(L(H))$ is Hamiltonian.

Other results about Eulerian and Hamiltonian dihypergraphs can be found in $[25]$.

\section{5 de Bruijn and Kautz Dihypergraphs}

In this section, we study the Eulerian and Hamiltonian properties of the generalization of de Bruijn and Kautz digraphs to dihypergraphs.

\section{1 de Bruijn, Kautz and Consecutive- $d$ digraphs}

First, we recall some definitions and previous results on digraphs that we will use in the sequel.

Definition 5 ([14, 15]). The generalized de Bruijn digraph $G B(d, n)$ (also called Reddy-Pradhan-Khul digraph), is the digraph whose vertices are labeled with the integers modulo $n$; there is an arc from vertex $i$ to vertex $j$ if, and only if, $j \equiv d i+\alpha(\bmod n)$, for every $\alpha$ with $0 \leq \alpha \leq d-1$.

If $n=d^{D}, G B(d, n)$ is nothing else than the de Bruijn digraph $B(d, D)$ (see $[13,24])$.

Definition 6 ([14]). The generalized Kautz digraph $G K(d, n)$ (also called Imase-Itoh digraph), is the digraph whose vertices are labeled with the integers modulo $n$; there is an arc from vertex $i$ to vertex $j$ if, and only if, $j \equiv$ $-d i-d+\alpha(\bmod n)$, for every $\alpha$ with $0 \leq \alpha \leq d-1$.

If $n=d^{D}+d^{D-1}, G K(d, n)$ is nothing else than the Kautz digraph $K(d, D)$ (see $[13,24])$.

Both of those families of digraphs can be generalized in the following way:

Definition 7 ([26]). Let $1 \leq d, q \leq n-1$, and $0 \leq r \leq n-1$, then the Consecutive- $d$ digraph $G(d, n, q, r)$ is the digraph whose vertices are labeled with the integers modulo $n$, such that there is an arc from vertex $i$ to vertex $j$ if, and only if, $j \equiv q i+r+\alpha(\bmod n)$, for every $\alpha$ with $0 \leq \alpha \leq d-1$. 
Observe that if $q=d$ and $r=0$, then $G(d, n, d, 0)=G B(d, n)$ and that if $q=r=n-d$, then $G(d, n, n-d, n-d)=G K(d, n)$.

Definition 8 ([27]). Let $\lambda$ be a positive integer, with $1 \leq \lambda \leq d$. Then $G B_{\lambda}(d, n)$ is the subdigraph of $G B(d, n)$ such that there is a link from $i$ to $j$ if, and only if, $j \equiv d i+\alpha(\bmod n)$, for every $0 \leq \alpha \leq \lambda-1$.

Actually, the digraph $G B_{\lambda}(d, n)$ is nothing else than the Consecutive- $d$ digraph $G(\lambda, n, d, 0)$. But the notation of $G B_{\lambda}(d, n)$ helps to understand that it is a subdigraph of $G B(d, n)$. If $\lambda=d, G B_{d}(d, n)=G B(d, n)$. We can define in a similar way $G K_{\lambda}(d, n)$.

Consecutive- $d$ digraphs have been intensively studied (see $[26,28,29,30,31$, $32,33,34,35])$. Particularly, the characterization of the Hamiltonian Consecutive$d$ digraphs is nearly complete:

Theorem $17([31,26,28,29])$. Let $G=G(d, n, q, r)$ be a Consecutive-d digraph.

- If $d=1$, then $G$ is Hamiltonian if, and only if, all of the four following conditions hold:

1. $\operatorname{gcd}(n, q)=1$;

2. for every prime number $p$ such that $p$ divides $n$, then we have $p$ divides $(q-1)$;

3. if 4 divides $n$, then 4 also divides $(q-1)$;

4. $\operatorname{gcd}(n, q-1, r)=1$.

- If $d=2$, then $G$ is Hamiltonian if, and only if, one of the following conditions is verified:

1. $\operatorname{gcd}(n, q)=2$;

2. $\operatorname{gcd}(n, q)=1$ and either $G(1, n, q, r)$ or $G(1, n, q, r+1)$ is Hamiltonian.

- If $d=3$, then:

1. if $\operatorname{gcd}(n, q) \geq 2, G$ is Hamiltonian if, and only if, $\operatorname{gcd}(n, q) \leq 3$;

2. if $\operatorname{gcd}(n, q)=1$ and $1 \leq|q| \leq 3, G$ is Hamiltonian.

- If $d \geq 4$, then $G$ is Hamiltonian if, and only if, $\operatorname{gcd}(n, q) \leq d$.

Corollary $2([28])$. Let $G=G(d, n, q, r)$ be a Consecutive-d digraph. If $\operatorname{gcd}(n, q) \geq$ 2 , then $G$ is Hamiltonian if, and only if, $\operatorname{gcd}(n, q) \leq d$.

The only remaining case is when $d=3$, for which there is only a partial characterization.

In particular, the characterization of the Hamiltonian generalized de Bruijn (resp. Kautz) digraphs is complete: 
Theorem $18([27])$. If $\lambda=\operatorname{gcd}(n, d) \geq 2$, then $G B_{\lambda}(d, n)$ and $G K_{\lambda}(d, n)$ are Hamiltonian.

Theorem 19 ([28, 27]). $G B(d, n)$ is Hamiltonian if, and only if, one of the following conditions holds:

1. $d \geq 3$;

2. $d=2$ and $n$ is even.

Theorem $20([28,27]) . G K(d, n)$ is Hamiltonian if, and only if, one of the following conditions holds:

1. $d \geq 3$;

2. $d=2$ and $n$ is even;

3. $d=2$ and $n$ is a power of 3 .

$G B(d, n)$ and $G K(d, n)$ are also Eulerian [27].

Finally, $G B(d, n)$ and $G K(d, n)$ have interesting line digraph properties. We use the following relations:

Proposition $21([27])$. If $\operatorname{gcd}(n, d)=\lambda \geq 2$, then

$$
L\left(G B_{\lambda}\left(d, \frac{n}{\lambda}\right)\right)=G B_{\lambda}(d, n) \text { and } L\left(G K_{\lambda}\left(d, \frac{n}{\lambda}\right)\right)=G K_{\lambda}(d, n)
$$

Particularly:

$$
L(G B(d, n))=G B(d, d n) \text { and } L(G K(d, n))=G K(d, d n) .
$$

\subsection{Definitions of de Bruijn and Kautz dihypergraphs}

We now give the arithmetical definition for the de Bruijn and Kautz dihypergraphs. For other definitions, see [12]. In what follows, the vertices (resp. the hyperarcs) are labeled with the integers modulo $n$ (resp. modulo $m$ ); the vertices are denoted $i, 0 \leq i \leq n-1$ and the hyperarcs $E_{j}, 0 \leq j \leq m-1$. To ease the reading we do not write, when it is clear in the context, the expressions $(\bmod n)$ and $(\bmod m)$.

Definition 9 ([12]). Let $d, n, s$ and $m$ be four positive integers, such that $d n \equiv 0(\bmod m)$ and $s m \equiv 0(\bmod n)$. The generalized de Bruijn dihypergraph $G B H(d, n, s, m)$ has as vertex set (resp. hyperarc set) the integers modulo $n$ (resp. modulo $m)$. Any vertex $i$ belongs to the in-set of hyperarcs $E_{d i+\alpha}(\bmod m)$, for every $0 \leq \alpha \leq d-1$. Any hyperarc $E_{j}$ has as out-set the vertices $s j+\beta$ $(\bmod n)$, for every $0 \leq \beta \leq s-1$.

Note that the condition $d n \equiv 0(\bmod m)$ follows from the fact that the vertices $i$ and $i+n$ should be incident to the same hyperarcs $d(i+n)+\alpha \equiv d i+\alpha$ $(\bmod m)$. Similarly $E_{j}^{+}=E_{j+m}^{+}$implies $s m \equiv 0(\bmod n)$. 
Particularly, when $n=m$, it can be useful to remark that in the bipartite digraph $R(G B H(d, n, s, n))$, the incidence relations from $\mathcal{V}_{1}$ to $\mathcal{V}_{2}$ are the same as in $G B(d, n)$ and the incidence relations from $\mathcal{V}_{2}$ to $\mathcal{V}_{1}$ are the same as in $G B(s, n)$.

Definition $10([12])$. Let $(d, n, s, m)$ be four positive integers, such that $d n \equiv 0$ $(\bmod m)$ and $s m \equiv 0(\bmod n)$. The generalized Kautz dihypergraph, denoted by $G K H(d, n, s, m)$, is the dihypergraph whose vertices (resp. hyperarcs) are labeled by the integers modulo $n$ (resp. modulo $m$ ), such that a vertex $i$ is incident to hyperarcs $E_{d i+\alpha(\bmod m)}$, for every $0 \leq \alpha \leq d-1$ and hyperarc $E_{j}$ has for out-set the vertices $-s j-s+\beta(\bmod n)$, for every $0 \leq \beta \leq s-1$.

By inversing the labeling of the hyperarcs, it has been proposed in [12] an equivalent definition for Kautz dihypergraphs:

Definition $11([12])$. Let $(d, n, s, m)$ be four positive integers, such that $d n \equiv 0$ $(\bmod m)$ and $s m \equiv 0(\bmod n)$. The generalized Kautz dihypergraph, denoted by $G K H(d, n, s, m)$, is the dihypergraph whose vertices (resp. hyperarcs) are labeled by the integers modulo $n$ (resp. modulo $m$ ), such that a vertex $i$ is incident to hyperarcs $E_{-d i-d+\alpha(\bmod m)}$, for every $0 \leq \alpha \leq d-1$ and hyperarc $E_{j}$ has for out-set the vertices $s j+\beta(\bmod n)$, for every $0 \leq \beta \leq s-1$.

We recall some properties that will be used in Section 6 .

Theorem 22 ([12]). The underlying multidigraph of $G B H(d, n, s, m)$ (resp. $G K H(d, n, s, m))$ is $G B(d s, n)$ (resp. $G K(d s, n))$.

Theorem 23 ([12]). If $H=G B H(d, n, s, m)$ (resp. $G K H(d, n, s, m)$ ), then $H^{*}=G B H(s, m, d, n)$ (resp. $\left.G K H(s, m, d, n)\right)$.

Theorem $24([19])$. The line dihypergraph of $G B H(d, n, s, m)$ (resp. of $G K H(d, n, s, m)$ ) is $G B H(d, d s n, s, d s m)$ (resp. is $G K H(d, d s n, s, d s m))$.

\subsection{Eulerian and Hamiltonian properties}

We now characterize when the generalized de Bruijn and Kautz dihypergraphs are Hamiltonian and Eulerian. Recall that we suppose $n>1$ and $m>1$.

Theorem 25. Let $H=G B H(d, n, s, m)$ be a generalized de Bruijn dihypergraph. $H$ is Hamiltonian if, and only if, one of the following conditions is verified:

1. $d s \geq 3$;

2. $d s=2$ and $n$ is even.

Proof. First, recall that $U(H)=G B(d s, n)$ by Theorem 22. By Theorem 19, we know that the de Bruijn digraph $G B(d s, n)$ is Hamiltonian if, and only if, $d s \geq 3$; or $d s=2$ and $n$ is even. Therefore, by Proposition 8 , Theorem 25 follows. 
Theorem 26. Let $H=G B H(d, n, s, m)$ be a generalized de Bruijn dihypergraph. $H$ is Eulerian if, and only if, one of the following conditions is verified:

1. $d s \geq 3$;

2. $d s=2$ and $m$ is even.

Proof. By Theorem 23, $H^{*}=G B H(s, m, d, n)$. Theorem 25 gives a necessary and sufficient condition for $H^{*}$ to be Hamiltonian. By Proposition 6, this is also a necessary and sufficient condition for $H$ to be Eulerian.

The method that is used for deciding whether $G B H(d, n, s, m)$ is Eulerian or Hamiltonian can be applied to Kautz dihypergraphs in the same way. By Theorem 20, we have necessary and sufficient conditions for a generalized Kautz digraph to be Hamiltonian. Consequently:

Theorem 27. Let $H=G K H(d, n, s, m)$ be a generalized Kautz dihypergraph.

1. If $d s \geq 3$, then $H$ is Eulerian and Hamiltonian;

2. If $d s=2$, then $H$ is Eulerian (resp. Hamiltonian) if, and only if, $m$ (resp. $n)$ is even or a power of 3 .

\section{Existence of Complete Berge Dicycles}

In this section, we want to determine when there exists a complete Berge dicycle in $G B H(d, n, s, m)$, (i.e a Hamiltonian dicycle in its bipartite representation digraph).

A necessary condition for a dihypergraph $H$ to have a complete Berge dicycle is that $n=m$. Otherwise, $R(H)$ cannot be Hamiltonian. We prove that:

Theorem 28. There is a complete Berge dicycle in $G B H(d, n, s, n)$ if one of the following conditions is verified:

1. $d \geq 3$ and $s \geq 3$;

2. $d=2$ and $s \geq 4$, or $s=2$ and $d \geq 4$;

3. $\{d, s\}=\{2,3\}$ and either $n$ is even or $n$ is a multiple of 3 ;

4. $d=s=2$ and $n$ is even or $n$ is a power of 3 (otherwise it does not exist);

5. $d=1$ or $s=1$ and $G B(d s, n)$ is Hamiltonian (otherwise it does not exist).

The only remaining case is when $\{d, s\}=\{2,3\}$ and $n$ and 6 are relatively prime, for which we conjecture $G B H(d, n, s, n)$ has a complete Berge dicycle:

Conjecture 29. If $\{d, s\}=\{2,3\}$, then there is a complete Berge dicycle in $G B H(d, n, s, n)$. 
We highlight the particular case when $s=d$, for which we have a complete characterization:

Theorem 30. There is a complete Berge dicycle in $G B H(d, n, d, n)$ if, and only if, one of the following conditions is verified:

1. $d \geq 3$;

2. $d=2$ and $n$ is an even number;

3. $d=2$ and $n$ is a power of 3 .

Remark that, for $d \geq 2$, these conditions are exactly the same as those implying that $G K(d, n)$ is Hamiltonian (see Theorem 20). It would be interesting to see if there is a relationship between Theorems 20 and 30 . We were able to find it only when $n$ is odd (see Lemma 5 in Section 6.4).

The rest of this section is devoted to the proof of Theorems 28 and 30. In Section 6.1, we deal with the easy case $d=1$. In Section 6.2, we show that Theorem 28 is true when $\operatorname{gcd}(n, d) \geq 2$ and $\operatorname{gcd}(n, s) \geq 2$ using a special product of digraphs and the notion of line digraphs. Then, in Section 6.3, we consider the opposite case, where $\operatorname{gcd}(n, d)=1 \operatorname{or} \operatorname{gcd}(n, s)=1$, and solve all the cases except $\{d, s\}=\{2,3\}, d=s=2$ and $d=s=3$. Section 6.4 contains the lemma which shows the relation with the generalized Kautz digraphs, and that the conditions of Theorem 30 are sufficient for $d=s=2$ and $n$ is a power of 3. In Section 6.5, by using the Euler's function, we show that these conditions are also necessary for $d=s=2$. Finally, in Section 6.6, we deal with the remaining case: $d=s=3$ and $\operatorname{gcd}(n, 3)=1$ and we solve it using a link-interchange method.

\subsection{Case $d=1$}

Lemma 1. If $d=1$, then there is a complete Berge dicycle in $G B H(1, n, s, n)$ if, and only if, the de Bruijn digraph $G B(s, n)$ is Hamiltonian.

Proof. If $d=1$, then every vertex $i$ is only incident to hyperarc $E_{i}$. So we may not distinguish the vertices from the hyperarcs and we get a digraph, the relations of incidence of which are the relations of incidence between hyperarcs and vertices in the original dihypergraph. Therefore, Lemma 1 follows.

By symmetry, observe that the case when $s=1$ is also solved by Lemma 1 .

\subsection{Case $\operatorname{gcd}(n, d) \geq 2$ and $\operatorname{gcd}(n, s) \geq 2$}

In this section, we completely solve the case when $\operatorname{gcd}(n, d) \geq 2$ and $\operatorname{gcd}(n, s) \geq$ 2. The proof is involved; in the particular case $d=s$, it can be simplified by using other methods such as the concatenation of digraph dicycles [25].

We will use a subcase of a digraph product introduced in [36, 37]: 
Definition 12 ([36, 37]). Let $L_{1}, L_{2}$ be two digraphs with the same order $n$ and with $\mathcal{V}\left(L_{1}\right) \cap \mathcal{V}\left(L_{2}\right)=\emptyset$ and let $\phi: \mathcal{V}\left(L_{1}\right) \rightarrow \mathcal{V}\left(L_{2}\right)$ be a one-to-one mapping. Then, $L_{1} \otimes_{\phi} L_{2}$ is the digraph $L$ such that $\mathcal{V}(L)=\mathcal{V}\left(L_{1}\right) \cup \mathcal{V}\left(L_{2}\right)$ and the set of arcs $\mathcal{E}(L)$ is defined by exchanging the out-neighbors of $u \in \mathcal{V}\left(L_{1}\right)$ with the out-neighbors of $\phi(u) \in \mathcal{V}\left(L_{2}\right)$ and vice-versa. More precisely, if $u_{2}=\phi\left(u_{1}\right)$, and $\left(u_{1}, v_{1}\right)$ is an arc of $L_{1}$ and $\left(u_{2}, v_{2}\right)$ is an arc of $L_{2}$, then the arcs $\left(u_{1}, v_{2}\right)$ and $\left(u_{2}, v_{1}\right)$ belong to $\mathcal{E}(L)$.

Observe that if $L_{1}$ is the generalized de Bruijn digraph $G B(s, n), L_{2}$ is the generalized de Bruijn digraph $G B(d, n)$ and $\phi$ is the identity function, then $L_{1} \otimes_{\phi} L_{2}$ is the bipartite representation digraph $R(G B H(d, n, s, n))$.

It happens that even if $L_{1}$ and $L_{2}$ are both strongly connected, $L_{1} \otimes_{\phi} L_{2}$ may be disconnected. However, it was proven by Barth and Heydemann the following sufficient condition:

Lemma 2 ([36]). If $L_{1}$ and $L_{2}$ are strongly connected, and if there exist $u_{1}$ and $u_{2}$ such that $\phi\left(u_{1}\right)=u_{2}$ and there is a loop $\left(u_{1}, u_{1}\right) \in \mathcal{E}\left(L_{1}\right)$ and a loop $\left(u_{2}, u_{2}\right) \in \mathcal{E}\left(L_{2}\right)$, then $L_{1} \otimes_{\phi} L_{2}$ is strongly connected.

We now prove a useful lemma:

Lemma 3. For every $i \in\{1,2\}$, let $D_{i}$ be an arbitrary digraph and $L_{i}=$ $L\left(D_{i}\right)$ be its line digraph. If $L_{1}$ and $L_{2}$ have the same number of vertices and $\phi: \mathcal{V}\left(L_{1}\right) \rightarrow \mathcal{V}\left(L_{2}\right)$ is a one-to-one mapping, then $L_{1} \otimes_{\phi} L_{2}$ is also a line multidigraph $L(D)$, such that $\mathcal{V}(D)=\mathcal{V}\left(D_{1}\right) \cup \mathcal{V}\left(D_{2}\right)$ and the degree of a vertex in $D$ is the same as the degree of its corresponding vertex in $D_{1}$ or $D_{2}$.

Proof. The vertices of $L_{i}(i=1,2)$ are the arcs of $D_{i}$ and so they are of the form $\left(u_{i}, v_{i}\right)$, with $u_{i}, v_{i} \in \mathcal{V}\left(D_{i}\right)$. Let $\mathcal{V}(D)=\mathcal{V}\left(D_{1}\right) \cup \mathcal{V}\left(D_{2}\right)$. For each $\left(u_{1}, v_{1}\right)$ of $L_{1}$, if $\left(u_{2}, v_{2}\right)=\phi\left(\left(u_{1}, v_{1}\right)\right)$ is its image by $\phi$, we put in $D$ the $\operatorname{arcs}\left(u_{1}, v_{2}\right)$ and $\left(u_{2}, v_{1}\right)$.

Now, consider the mapping $\psi: \mathcal{V}\left(L_{1} \otimes_{\phi} L_{2}\right) \rightarrow \mathcal{V}(L(D))=\mathcal{E}(D)$, defined as follows: if $\left(u_{1}, v_{1}\right)$ is a vertex of $L_{1}$ and $\left(u_{2}, v_{2}\right)=\phi\left(\left(u_{1}, v_{1}\right)\right)$ is the associated vertex in $L_{2}$, then $\psi\left(\left(u_{1}, v_{1}\right)\right)=\left(u_{1}, v_{2}\right)$ and $\psi\left(\left(u_{2}, v_{2}\right)\right)=\left(u_{2}, v_{1}\right)$. Observe that $\psi$ is a one-to-one mapping. To prove the lemma, it suffices to prove that $\psi$ keeps the adjacency relation.

On one side, by definition of the product, the vertex $\left(u_{1}, v_{1}\right)$ is joined in $L_{1} \otimes_{\phi} L_{2}$ to the out-neighbors of $\left(u_{2}, v_{2}\right)$ in $L_{2}$ that is to the vertices of the form $\left(v_{2}, w_{2}\right)$, with $\left(v_{2}, w_{2}\right)$ an arc of $D_{2}$. On the other side, in $L(D)$, the vertex $\left(u_{1}, v_{2}\right)=\psi\left(\left(u_{1}, v_{1}\right)\right)$ is joined to the vertices $\left(v_{2}, y_{1}\right)$, where $y_{1}$ is such that there exists $x_{1}$ in $D_{1}$ and $w_{2}$ in $D_{2}$, such that $\left(x_{1}, y_{1}\right)$ is an arc of $D_{1}, \phi\left(\left(x_{1}, y_{1}\right)\right)=$ $\left(v_{2}, w_{2}\right)$ and $\left(v_{2}, w_{2}\right)$ is an arc of $D_{2}$. But, by definition, $\left(v_{2}, y_{1}\right)=\psi\left(\left(v_{2}, w_{2}\right)\right)$. So, $\psi\left(\left(u_{1}, v_{1}\right)\right)$ is joined in $L(D)$ to all the images by $\psi$ of the out-neighbors of $\left(u_{1}, v_{1}\right)$ in $L_{1} \otimes_{\phi} L_{2}$ and then the adjacency relation is kept for the vertices of $L_{1}$. The proof is identical for the vertices of $L_{2}$.

When $s=d$, we can prove a stronger result namely that $G B_{\lambda}(d, n) \otimes_{\phi}$ $G B_{\lambda}(d, n)$ is the line digraph of $G B_{\lambda}\left(d, \frac{n}{\lambda}\right) \otimes_{\phi} G B_{\lambda}\left(d, \frac{n}{\lambda}\right)[25]$. 
Remark 4. Note that, if $L_{1}=L\left(D_{1}\right)$ and $L_{2}=L\left(D_{2}\right)$ are Hamiltonian digraphs, then $D_{1}$ and $D_{2}$ are balanced and so, by Lemma 3, $D$ is a balanced digraph, i.e. every vertex of $D$ has equal in-degree and out-degree.

Lemmas 2 and 3 enable us to prove the following theorem:

Theorem 31. Let $H=G B H(d, n, s, n)$ be a generalized de Bruijn dihypergraph. If $\operatorname{gcd}(d, n) \geq 2$ and $\operatorname{gcd}(s, n) \geq 2$, then there is a complete Berge dicycle in $H$.

Proof. Let us show that $R=R(G B H(d, n, s, n))$ is a Hamiltonian digraph. We recall that $R$ is isomorphic to $G B(s, n) \otimes_{\phi} G B(d, n), \phi$ being the identity function. So, for $\lambda=\operatorname{gcd}(d, n)$ and $\mu=\operatorname{gcd}(s, n)$, the digraph $G B_{\mu}(s, n) \otimes_{\phi}$ $G B_{\lambda}(d, n)$ is isomorphic to a subdigraph of $R$. As, by Proposition $21, G B_{\mu}(s, n)$ and $G B_{\lambda}(d, n)$ are two line digraphs, then, by Lemma $3, G B_{\mu}(s, n) \otimes_{\phi} G B_{\lambda}(d, n)$ is also a line digraph $L(D)$. Moreover, since $G B_{\mu}(s, n)$ and $G B_{\lambda}(d, n)$ are also Hamiltonian digraphs, by Theorem 18 , then $D$ is a balanced digraph by Remark 4. Furthermore, both $G B_{\mu}(s, n)$ and $G B_{\lambda}(d, n)$ are strongly connected and those two digraphs have a common loop $(0,0)$. Consequently, by Lemma $2, G B_{\mu}(s, n) \otimes_{\phi} G B_{\lambda}(d, n)$ is strongly connected, hence $D$ is strongly connected too.

$D$ is a balanced digraph that is strongly connected. In other words, $D$ is an Eulerian digraph and so $L(D)=G B_{\mu}(s, n) \otimes_{\phi} G B_{\lambda}(d, n)$ is a Hamiltonian digraph.

\subsection{Case $n$ and $d$ relatively prime, or $n$ and $s$ relatively prime}

In the next proofs, we consider a Hamiltonian dicycle in a Consecutive- $d$ digraph as a circular permutation $\sigma$ in $\mathbb{Z}_{n}$. If $j$ is the vertex that follows $i$ in the Hamiltonian dicycle, then $\sigma(i)=j$; if $k$ is the vertex that follows $j$ in the same dicycle, then $\sigma^{2}(i)=k$ and so on.

Now we deal with the other case $\operatorname{gcd}(n, d)=1$ or $\operatorname{gcd}(n, s)=1$ and will prove that the Theorem 28 holds in most of the cases. The proof will rely on the following lemma:

Lemma 4. Let $n$ and $d$ be relatively prime. If the Consecutive-s digraph $G(s, n, d s, 0)$ is Hamiltonian, then there is a complete Berge dicycle in $G B H(d, n, s, n)$.

Proof. Recall that in $G(s, n, d s, 0)$ a vertex $i$ is joined to the vertices $j \equiv d s i+\beta$ $(\bmod n)$, for every $\beta$ with $0 \leq \beta \leq s-1$. Let $0, \sigma(0), \sigma^{2}(0), \ldots, \sigma^{n-1}(0), 0$ be a Hamiltonian dicycle of $G(s, n, d s, 0)$. We construct the following dicycle in $G B H(d, n, s, n)$. Vertex $i$ precedes the hyperarc $E_{d i}$. Since $\operatorname{gcd}(n, d)=1$, therefore $d$ is invertible in $Z_{n}$ and $i \rightarrow d i$ is a bijection between vertices and hyperarcs. The dicycle $0, E_{0}, \sigma(0), E_{d \sigma(0)}, \ldots, \sigma^{n-1}(0), E_{d \sigma^{n-1}(0)}, 0$ is then a complete Berge dicycle in $G B H(d, n, s, n)$; indeed the vertex $\sigma^{k+1}(0) \equiv s\left(d \sigma^{k}(0)\right)+\beta_{k} \equiv$ $s\left(d \sigma^{k}(0)\right)+\beta_{k}$ is in the out-set of $E_{d \sigma^{k}(0)}$. 
Theorem 32. Let $H=G B H(d, n, s, n)$ be a generalized de Bruijn dihypergraph such that $d \neq 1$ and $s \neq 1$. If $n$ and $d$ are relatively prime or $n$ and $s$ are relatively prime, then there is a complete Berge dicycle in $H$ if one of the following conditions hold:

1. $d \geq 4$ or $s \geq 4$;

2. $\{d, s\}=\{2,3\}$ and $n$ is even or $n$ is a multiple of 3 .

Proof. By Theorem 17, we know that $G(s, n, d s, 0)$ is Hamiltonian if one of the following conditions hold:

- $s \geq 4$ and $\operatorname{gcd}(n, d s) \leq s$;

- or $\{s=3$ and $2 \leq \operatorname{gcd}(n, 3 d) \leq 3\}$;

- or $\{s=2$ and $\operatorname{gcd}(n, 2 d)=2\}$.

Furthermore, if $n$ and $d$ are relatively prime, we have:

$$
\operatorname{gcd}(n, d s)=\operatorname{gcd}(n, s) \leq s
$$

and so, $2 \leq \operatorname{gcd}(n, 3 d) \leq 3$ is equivalent to $n$ multiple of 3 and $\operatorname{gcd}(n, 2 d)=2$ is equivalent to $n$ even.

By using these facts and Lemma 4 we get:

- Fact 1: if $n$ and $d$ are relatively prime, then there is a complete Berge dicycle in $G B H(d, n, s, n)$ when $s \geq 4$ or $\{s=3$ and $n$ is a multiple of 3$\}$ or $\{s=2$ and $n$ is even $\}$.

- Fact 2: (obtained by exchanging $d$ and $s$ ) if $n$ and $s$ are relatively prime, then there is a complete Berge dicycle in $\operatorname{GBH}(s, n, d, n)$, hence, there is also a complete Berge dicycle in the dual $G B H(d, n, s, n)$, when $d \geq 4$ or $\{d=3$ and $n$ is a multiple of 3$\}$ or $\{d=2$ and $n$ is even $\}$.

Now, we can conclude as follows:

Let $d \geq 4$. If $n$ and $s$ are relatively prime we conclude by using Fact 2 . Otherwise $\operatorname{gcd}(n, s) \geq 2$ and $n$ and $d$ are relatively prime. The theorem is proved by using Fact 1 as either $s \geq 4$; or $s=3$, but then $n$ is a multiple of 3 , because $\operatorname{gcd}(n, s) \geq 2$; or $s=2$ and $\operatorname{gcd}(n, s) \geq 2$ implies that $n$ is a multiple of 2.

The case $s \geq 4$ can be done similarly (by exchanging $d$ and $s$, which corresponds to work in the dual).

Now let $d=3$ and $s=2$. If $n$ is a multiple of $d=3$, then by hypothesis $n$ and $s$ are relatively prime and we conclude by Fact 2. If $n$ is a multiple of $s=2$, then by hypothesis $n$ and $d$ are relatively prime and we conclude by Fact 1. The case $d=2$ and $s=3$ is done similarly by exchanging $d$ and $s$. 


\subsection{Concatenation of dicycles and relation to generalized Kautz digraphs}

If $n$ is an odd number, then Theorem 30 can be partly proven with a concatenation of dicycles.

Lemma 5. If $G K(d, n)$ is Hamiltonian and $n$ is odd, then there is a complete Berge dicycle in $G B H(d, n, d, n)$.

Proof. We use a variant for the definition of $G B H(d, n, d, n)$. Indeed as noted in [38], if we label the hyperarc $E_{j}$ with label $E_{n-1-j}$, we get the incidence relations of $G K(d, n)$. In other words, $G B H(d, n, d, n)$ can be defined as follows: vertex $i$ is incident to hyperarcs $E_{-d i-d+\alpha}(\bmod n)$, for every $0 \leq \alpha \leq d-1$, and hyperarc $E_{j}$ has as out-set the vertices $-d j-d+\beta(\bmod n)$, for every $0 \leq \beta \leq d-1$.

Now, by Theorem 20, there exists a Hamiltonian dicycle in the Kautz digraph $G K(d, n)$ for $n$ odd, and either $d \geq 3$ or $\{d=2$ and $n$ is a power of 3$\}$; let it be $0, \sigma(0), \sigma^{2}(0), \ldots, \sigma^{n-1}(0), 0$. Let $C$ be the dicycle of $G B H(d, n, d, n)$, where vertex $i$ precedes hyperarc $E_{\sigma(i)}$ and hyperarc $E_{j}$ precedes vertex $\sigma(j)$. So, $C=0, E_{\sigma(0)}, \sigma^{2}(0), \ldots, \sigma^{2 h}(0), E_{\sigma^{2 h+1}(0)}, \ldots, \sigma^{n-2}(0), E_{\sigma^{n-1}(0)}, 0$, where $0 \leq$ $h \leq n-1$. As $n$ is odd, the $n$ vertices and also the $n$ hyperarcs of the dicycle are all different. Therefore $C$ is a complete Berge dicycle in $G B H(d, n, d, n)$.

Corollary 3. If $n$ is odd and $d \geq 3$, then there is a complete Berge dicycle $C$ in $G B H(d, n, d, n)$.

Corollary 4. If $n$ is a power of 3 and $d=2$, then there is a complete Berge dicycle $C$ in $G B H(2, n, 2, n)$.

In the same way, we can prove that, if $G B(d, n)$ is Hamiltonian and $n$ is odd, then there is a complete Berge dicycle in $G B H(d, n, d, n)$. However, even if it would have given the result for $G B H(d, n, d, n)$ with $n$ odd and $d \geq 3$, it would have not been enough to conclude for the case $d=2$ and $n$ a power of 3 . In that case, the proof of Lemma 5 plus the fact that, by [28], $\sigma: i \rightarrow-2 i-1$ is a Hamiltonian dicycle in $G K(2, n)$, gives the following complete Berge dicycle $C$ in $G B H(2, n, 2, n)$ (by renaming the edges with the standard definition). In $C$, vertex $i$ precedes the hyperarc $E_{2 i}$, and hyperarc $E_{j}$ precedes the vertex $2 j+1$. Thus, $C$ contains as consecutive vertices $i$ and $4 i+1$. Figure 1 shows the dicycle $0, E_{0}, 1, E_{2}, 5, E_{1}, 3, E_{6}, 4, E_{8}, 8, E_{7}, 6, E_{3}, 7, E_{5}, 2, E_{4}, 0$, that is obtained in this way for $n=9$ (with dotted red arcs).

\subsection{Case $d=s=2$}

Theorem 31 and Corollary 4 show that there exists a complete Berge dicycle in $\operatorname{GBH}(2, n, 2, n)$ when $n$ is even, or $\{n$ is odd and $n$ is a power of 3$\}$. We still have to prove there is no complete Berge dicycle in the remaining cases. For that, we need to use the Euler function, in the spirit of the proof of [28]. 
Definition 13. The Euler function, denoted by $\varphi$, associates to a positive integer $n$, the number $\varphi(n)$ of positive integers that are lower than $n$ and relatively prime to $n$.

The Euler function satisfies the following properties (the three first ones are immediate consequences of the definition and the fourth one is known as Euler's theorem):

1. $\varphi(1)=1$;

2. If $p$ is a prime number and $m \geq 1$, then $\varphi\left(p^{m}\right)=(p-1) p^{m-1}$;

3. If $a$ and $b$ are relatively prime, then $\varphi(a b)=\varphi(a) \varphi(b)$;

4. If $a$ and $b$ are relatively prime, then $a^{\varphi(b)} \equiv 1(\bmod b)$.

Lemma 6. If $n$ is odd, then there is a complete Berge dicycle in $G B H(2, n, 2, n)$ if, and only if, $n$ is a power of 3 .

Proof. Let us suppose that there is a complete Berge dicycle $C$ in $G B H(2, n, 2, n)$. We distinguish two cases: either there exists a vertex $i$, such that $i$ precedes in $C$ the hyperarc $E_{2 i}$ and we will show this holds for all the vertices; or any vertex $i$ precedes in $C$ the hyperarc $E_{2 i+1}$. To prove this claim, consider that some vertex $i$ precedes $E_{2 i}$ in $C$. Since $\operatorname{gcd}(2, n)=1$, we have that 2 is invertible in $\mathbb{Z}_{n}$. Thus, $i^{\prime}=i-2^{-1}$ cannot precede $E_{2 i^{\prime}+1}$, as $2 i^{\prime}+1=2 i$. So, $i^{\prime}$ precedes $E_{2 i^{\prime}}$ too. Consequently, since $2^{-1}$ is a generator element of $\mathbb{Z}_{n}$, any vertex $i$ precedes in $C$ the hyperarc $E_{2 i}$.

Similarly, we can prove that either every hyperarc $E_{j}$ in $C$ precedes the vertex $2 j$, or every hyperarc $E_{j}$ in $C$ precedes the vertex $2 j+1$. Therefore, if we consider only the vertices of the dicycle $C$ and we denote by $\sigma(i)$ the successor of $i$ in $C$, we have exactly four possibilities for $\sigma$, namely: $\sigma_{k}(i)=4 i+k$ with $0 \leq k \leq 3$.

Since $4 \cdot 0=0$, the solution $k=0$ does not generate a complete Berge dicycle. Furthermore, if $\operatorname{gcd}(n, 3)=1$ the equation $\sigma_{k}(i) \equiv i \Longleftrightarrow 4 i+k \equiv i$ $\Longleftrightarrow 3 i \equiv-k$, has always a solution for $1 \leq k \leq 3$. Therefore, none of the other values of $k$ works, when $\operatorname{gcd}(n, 3)=1$.

It remains to consider the case $n=c 3^{p}, p \geq 1$ and $\operatorname{gcd}(3, c)=1$. By induction, we have that $\sigma_{k}^{h}(0)=\frac{k\left(4^{h}-1\right)}{3}(\bmod n)$.

Let $\varphi$ be the Euler function; by Properties 2 and 3, $\varphi(n)=\varphi(c) \varphi\left(3^{p}\right)=$ $2 \varphi(c) 3^{p-1}$. Since $\operatorname{gcd}(n, 2)=1$, then, by Property $4,2^{\varphi(n)} \equiv 1(\bmod n)$. Therefore, $4^{\varphi(c) 3^{p-1}} \equiv 1(\bmod n)$ and, since $\varphi(c) 3^{p-1}<n, \sigma_{3}$ never generates a complete Berge dicycle either.

Moreover, since 2 is invertible in $\mathbb{Z}_{n}$, then we also know that $\sigma_{2}$ generates a complete Berge dicycle if, and only if, this is also the case for solution $\sigma_{1}$. Actually, when we choose $\sigma_{1}$, we choose $\sigma_{2}$ in the dual, and reciprocally. So, let us concentrate now on $\sigma_{1}$. The equation $\sigma_{1}^{h}(0)=\sigma_{1}^{h^{\prime}}(0)$ is equivalent to $4^{h} \equiv 4^{h^{\prime}}(\bmod 3 n)$. Again, Property 4 of Euler's function implies that $4^{\varphi(c) 3^{p}} \equiv$ 
$1(\bmod 3 n)$. But the only value of $c$ such that $\varphi(c)=c$ is $c=1$. Therefore, if $n$ is not a power of $3, \sigma_{1}$ and $\sigma_{2}$ do not generate a complete Berge dicycle.

This proof for $d=2$ and $n$ odd could be shortened using the characterization of the Hamiltonian Consecutive-1 digraphs. Indeed, we prove there are only four possibilities for having a complete Berge dicycle in $G B H(2, n, 2, n)$. In the original proof, we deal with them as applications $\sigma_{k}$ of $\mathbb{Z}_{n}$, for $0 \leq k \leq 3$. But these four solutions are also equivalent to some Consecutive- 1 digraphs. They correspond, respectively, to the relations of incidence in $G(1, n, 4,0), G(1, n, 4,1)$, $G(1, n, 4,2)$ and $G(1, n, 4,3)$. Then, deciding whether one of these four solutions generate a complete Berge dicycle is the same thing as deciding whether one of these four Consecutive-1 digraphs is a Hamiltonian digraph. Furthermore, by Theorem 17, we know whether one of those digraphs is Hamiltonian, depending on the value of $n$. For all $n, G(1, n, 4,0)$ and $G(1, n, 4,3)$ are never Hamiltonian. Moreover, $G(1, n, 4,1)$ and $G(1, n, 4,2)$ are Hamiltonian if, and only if, $n$ is a power of 3 . Since the Hamiltonicity of at least one of these digraphs is a necessary and sufficient condition for $H=G B H(2, n, 2, n)$ to have a complete Berge dicycle, then there is a complete Berge dicycle in $H$ if, and only if, $n$ is a power of 3 .

\subsection{Case $d=s=3$}

To finish the proof, it remains to deal with the case $n$ even, $d=s=3$ and $n$ and $d$ relatively prime. Note that, for $d=3$, we do not know when the Consecutive- 3 digraph $G(3, n, 9,0)$ is Hamiltonian, and so, we cannot use the same proof as in Lemma 4. We will use a method, introduced in [28], that is different from the previous ones. This method enables us to merge two disjoint dicycles of $R(H)$ into one dicycle.

Definition 14. Let $C_{1}, C_{2}$ be two dicycles, that are subdigraphs of the same digraph D. A pair $\left\{x_{1}, x_{2}\right\}$ with $x_{1} \in C_{1}$ and $x_{2} \in C_{2}$ is called an interchange pair if the predecessor $y_{1}$ of $x_{1}$ in $C_{1}$ is incident to $x_{2}$ in $D$, and the predecessor $y_{2}$ of $x_{2}$ in $C_{2}$ is incident to $x_{1}$ in $D$ too.

If $\left\{x_{1}, x_{2}\right\}$ is an interchange pair, then we can build a dicycle containing all the vertices of $C_{1} \cup C_{2}$ by deleting $\left(y_{1}, x_{1}\right)$ and $\left(y_{2}, x_{2}\right)$ and adding the arcs $\left(y_{1}, x_{2}\right)$ and $\left(y_{2}, x_{1}\right)$.

Lemma 7. If $n$ is even and $n$ and 3 are relatively prime, then there is a complete Berge dicycle in $G B H(3, n, 3, n)$.

Proof. Let $R$ be the bipartite representation digraph of $G B H(3, n, 3, n)$. To every vertex $i$ we associate the hyperarc $E_{3 i+1}$ and, similarly, to every hyperarc $E_{j}$ we associate the vertex $3 j+1$. Since $\operatorname{gcd}(n, 3)=1$, the digraph $R$ is partitioned into pairwise vertex-disjoint dicycles. If there is only one dicycle in this partition, we are done as it is Hamiltonian. Otherwise, we use interchange pairs to merge successively the dicycles till we have only one. But we have to be careful to do independent interchanges. 
Figure 4 shows an example for the case $n=8$, where we obtain the 4 dicycles: $C_{0}=\left(0, E_{1}, 4, E_{5}\right), C_{1}=\left(1, E_{4}, 5, E_{0}\right), C_{2}=\left(2, E_{7}, 6, E_{3}\right), C_{3}=\left(3, E_{2}, 7, E_{6}\right)$.

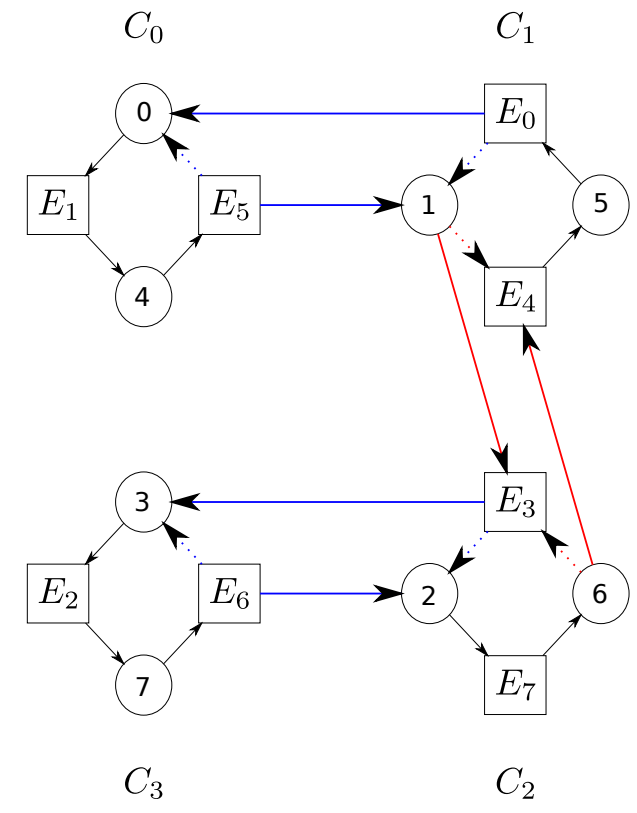

Figure 4: An application of the link-interchange method to the de Bruijn dihypergraph $G B H(3,8,3,8)$.

We first claim that, if $i$ and $i+1$ belong to two disjoint dicycles $C_{1}$ and $C_{2}$, then $\{i, i+1\}$ is an interchange pair. Indeed, let $E_{j}$ be the predecessor of $i$ in $C_{1}$ and $E_{j^{\prime}}$ the predecessor of $i+1$ in $C_{2}$. By construction, $3 j+1=i$ and $3 j^{\prime}+1=i+1$. Consequently, $3 j+2=i+1$ and so there is an arc from $E_{j}$ to $i+1$. We also have $3 j^{\prime}=i$ and so there is an arc from $E_{j}^{\prime}$ to $i$. Therefore, the claim is proved. For $n=8,\{0,1\}$ is an interchange pair and we can merge $C_{0}$ and $C_{1}$ by deleting the $\operatorname{arcs}\left(E_{5}, 0\right)$ and $\left(E_{0}, 1\right)$ (in dashed blue in Figure 4$)$ and adding the $\operatorname{arcs}\left(E_{5}, 1\right)$ and $\left(E_{0}, 0\right)$ (in blue).

Similarly, we have that if $E_{j}$ and $E_{j+1}$ belong to two disjoint dicycles then $\left\{E_{j}, E_{j+1}\right\}$ is an interchange pair. We have to be careful not to use twice the same vertex in an interchange pair, as the predecessor has changed when doing the first merging. Here we will use only some interchange pairs of the form $\{2 i, 2 i+1\}$ and $\left\{E_{2 j+1}, E_{2 j+2}\right\}$, which are pairwise independent because $n$ is even.

We proceed as follows: if there exists an $i$ such that $2 i$ and $2 i+1$ belong to different dicycles we merge these dicycles using the interchange pair $\{2 i, 2 i+1\}$. After at most $n / 2$ merge operations, we get a set of disjoint dicycles such that, for all i, $2 i$ and $2 i+1$ belong to the same dicycle. In the example for $n=8$, we merge $C_{0}$ and $C_{1}$ using the interchange pair $\{0,1\}$ and $C_{2}$ and $C_{3}$ using the 
interchange pair $\{2,3\}$ (see Figure 4 ). We now have 2 dicycles.

Then, consider two vertices of the form $2 i$ and $2\left(i+3^{-1}\right)$. Suppose that they belong to two different dicycles $C_{1}$ and $C_{2}$. The vertex $2 i+1$, which is also in $C_{1}$ precedes the hyperarc $E_{6 i+4}$ in $C_{1}$ and the vertex $2\left(i+3^{-1}\right)$ precedes $E_{6 i+3}$ in $C_{2}$. Moreover, we claim that $\left\{E_{6 i+3}, E_{6 i+4}\right\}$ is an admissible interchange pair that we can use to merge the two dicycles, because $6 i+3$ is odd whereas $n$ is even, and so, $6 i+3(\bmod n)$ is odd. Finally, since 3 and $n$ are relatively prime, $3^{-1}$ is a generator element in $\mathbb{Z}_{n}$ and so we can consider successively the possible $i$ such that $2 i$ and $2\left(i+3^{-1}\right)$ belong to two different dicycles and merge all the dicycles.

Observe that for the example in Figure 4, when $n=8$, we have that $3^{-1}=3$. We now use the construction for $i=0$. Vertices 0 and 6 are in two different dicycles, and $\left\{E_{3}, E_{4}\right\}$ is an admissible interchange pair. So we can merge the two dicycles by deleting the arcs $\left(6, E_{3}\right)$ and $\left(1, E_{0}\right)$ (in dashed red in Figure 4 ) and adding the arcs $\left(1, E_{3}\right)$ and $\left(6, E_{4}\right)$ (in red) to get the final complete Berge dicycle $C=0, E_{1}, 4, E_{5}, 1, E_{3}, 3, E_{2}, 7, E_{6}, 2, E_{7}, 6, E_{4}, 5, E_{0}, 0$.

\subsection{Complete Berge dicycles in Kautz Dihypergraphs}

The Kautz dihypergraph $G K H(d, n, d, n)$ is close to the dihypergraph $G B H(d, n, d, n)$, but the existence of complete Berge dicycles in it is much harder to prove due to its asymmetry. Indeed, the relations of incidence from its vertices to the hyperarcs are not the same as the relations of incidence from its hyperarcs to the vertices.

Nonetheless, we have been able to show the existence of complete Berge dicycles in $G K H(d, n, d, n)$ for some particular values of $(d, n)$. Remark that $R(G K H(d, n, d, n))$ is isomorphic to the bipartite digraph $B D(d, n)$ (see [39]). The proof of the following theorem uses the same tools as for $G B H(d, n, d, n)$ and can be found in $[25]$

Theorem 33. Let $H=G K H(d, n, d, n)$ be a Kautz dihypergraph. There is a complete Berge dicycle in $H$ if one of the following conditions is verified:

1. $d \geq 4$;

2. $d=3$ and $n$ is even;

3. $d=2$ and $n$ is even or $n$ is a power of 5 (otherwise it does not exist);

4. $d=1$ and $n \in\{1,2\}$ (otherwise it does not exist).

We also have the following conjecture concerning complete Berge dicycles in $G K H(d, n, d, n)$ :

Conjecture 34. Let $H=G K H(d, n, d, n)$ be a Kautz dihypergraph. If $d \geq 3$, then there is a complete Berge dicycle in $H$. 


\section{Conclusions}

In this paper, we showed that it is an NP-complete problem to decide whether a dihypergraph is Eulerian (or Hamiltonian). We presented a generalization of some results concerning Eulerian digraphs, in the case where the studied dihypergraphs are uniform and regular. Then, we studied the Eulerian and Hamiltonian properties of generalized de Bruijn and Kautz dihypergraphs.

We let as open questions Conjectures 29 and 34. It would also be nice to find a relationship between Theorems 20 and 30, since both have similar conditions and different implications.

\section{References}

[1] J. Bang-Jensen, G. Gutin, Digraphs: theory, algorithms and applications, Springer Verlag, 2010.

[2] J. Bondy, U. Murty, Graph Theory with applications, 2nd Edition, Vol. 290, MacMillan London, 2008.

[3] C. Berge, Graphs and hypergraphs, Vol. 6, North-Holland Pub. Co., 1973.

[4] Z. Lonc, P. Naroski, On tours that contain all edges of a hypergraph, The electronic journal of combinatorics 17 (R144) (2010) 1.

[5] J.-C. Bermond, Hamiltonian decompositions of graphs, directed graphs and hypergraphs, Ann. Discrete Math. 3 (1978) 21-28, présenté au Cambridge Combinatorial Conf., Advances in graph theory , Trinity College, Cambridge, England, 1977.

[6] P. Keevash, D. Kühn, R. Mycroft, D. Osthus, Loose Hamilton cycles in hypergraphs, Discrete Mathematics 311 (2011) 544-559.

[7] D. Kühn, R. Mycroft, D. Osthus, Hamilton l-cycles in uniform hypergraphs, J. Comb. Theory Ser. A 117 (2010) 910-927. doi:10.1016/j.jcta.2010.02.010.

[8] E. Arkin, M. Held, J. S. B. Mitchell, S. Skiena, Hamilton triangulations for fast rendering, in: Proceedings of the Second Annual European Symposium on Algorithms, ESA '94, Springer-Verlag, London, UK, 1994, pp. 36-47.

[9] J. Bartholdi III, P. Goldsman, Multiresolution indexing of triangulated irregular networks., IEEE Trans. Vis. Comput. Graph. 10 (2004) 484-495.

[10] J. Bartholdi III, P. Goldsman, The vertex-adjacency dual of a triangulated irregular network has a Hamiltonian tour, Operations Research Letters 32 (2004) 304-308.

[11] A. Dudek, A.Frieze, A. Ruciński, Rainbow hamilton cycles in uniform hypergraphs, Electronic Journal of Combinatorics 19 (2012) P46. 
[12] J.-C. Bermond, R. W. Dawes, F. Ergincan, De Bruijn and Kautz bus networks, Networks 30 (3) (1997) 205-218. doi:10.1002/(SICI)10970037(199710)30:3¡205::AID-NET5¿3.0.CO;2-P.

[13] J.-C. Bermond, C. Peyrat, De Bruijn and Kautz networks: a competitor for the hypercube?, in: F. André, J.-P. Verjus (Eds.), Proceedings of the 1st European Workshop on Hypercubes and Distributed Computers, Rennes, North Holland, 1989, pp. 279-293.

[14] M. Imase, M. Itoh, Design to minimize diameter on building-block network, IEEE Transactions Computers C-30 (1981) 439-442.

[15] S. Reddy, D. Pradhan, J. Kuhl, Directed graphs with minimal diameter and maximal connectivity, Tech. rep., Oakland University, School of Engineering (1980).

[16] D.-Z. Du, D. F. Hsu, F. K. Hwang, The Hamiltonian property of consecutive-d digraphs, Mathematical and Computer Modelling 17 (11) (1993) 61 - 63. doi:10.1016/0895-7177(93)90253-U.

[17] T. E. Stern, K. Bala, Multiwavelength Optical Networks: A Layered Approach, Addison-Wesley Longman Publishing Co., Inc., Boston, MA, USA, 1999.

[18] J.-C. Bermond, F. Ergincan, Bus interconnection networks, Discrete Applied Mathematics 68 (1996) 1-15.

[19] J.-C. Bermond, F. Ergincan, M. Syska, Quisquater Festschrift, Vol. 6805 of Lecture Notes in Computer Science, Springer-Verlag, Berlin Heidelberg, 2011, Ch. Line Directed Hypergraphs, pp. 25-34.

[20] D. Ferrero, C. Padró, Connectivity and fault-tolerance of hyperdigraphs, Discrete Applied Mathematics 117 (2002) 15-26.

[21] D. Ferrero, C. Padró, Partial line directed hypergraphs, Networks 39 (2002) 61-67.

[22] V. Batagelj, T. Pisanski, On partially directed Eulerian multigraphs, Publ. de l'Inst. Math. Soc. 25 (39) (1979) 16-24.

[23] J. Bang-Jensen, S. Thomassé, Decompositions and orientations of hypergraphs, Tech. Rep. 10, IMADA (2001).

[24] J. De Rumeur, Communications dans les réseaux de processeurs, Masson, 1994.

[25] G. Ducoffe, Eulerian and Hamiltonian directed hypergraphs, Research Report 7893, INRIA (2012).

[26] D.-Z. Du, D. Hsu, On Hamiltonian consecutive-d digraphs, Banach Center Publications 25 (1989) 47-55. 
[27] D.-Z. Du, F. K. Hwang, Generalized de Bruijn digraphs, Networks 18 (1) (1988) 27-38. doi:10.1002/net.3230180105.

[28] D.-Z. Du, D. F. Hsu, F. K. Hwang, X. M. Zhang, The Hamiltonian property of generalized de Bruijn digraphs, Journal of Combinatorial Theory, Series B 52 (1) (1991) 1 - 8. doi:DOI: 10.1016/0095-8956(91)90084-W.

[29] F. K. Hwang, The Hamiltonian property of linear functions, Operations Research Letters 6 (3) (1987) 125 - 127. doi:10.1016/0167-6377(87)900241 .

[30] G. Chang, F. Hwang, L.-D. Tong, The Hamiltonian property of the consecutive-3 digraph, Mathematical and Computer Modelling 25 (11) (1997) 83 - 88. doi:10.1016/S0895-7177(97)00086-1.

[31] G. Chang, F. Hwang, L.-D. Tong, The consecutive-4 digraphs are Hamiltonian, J. Graph Theory 31 (1999) 1-6.

[32] D.-Z. Du, D. Hsu, D. J. Kleitman, Modification of consecutive- $d$ digraphs., in: Interconnection Networks and Mapping and Scheduling Parallel Computations. Proceedings DIMACS workshop February, 1994 Rutgers University, New Brunswick, NJ, USA, Providence, RI: American Mathematical Society, 1994, pp. 75-85.

[33] D.-Z. Du, D. Hsu, G. Peck, Connectivity of consecutive-d digraphs, Discrete Appl. Math. 37-38 (1992) 169-177. doi:10.1016/0166-218X(92)90131-S.

[34] D.-Z. Du, D. Hsu, H. Ngo, G. Peck, On connectivity of consecutived digraphs, Discrete Math. 257 (2002) 371-384. doi:10.1016/S0012365X(02)00436-3.

[35] F. Cao, D.-Z. Du, F. Hsu, L. Hwang, W. Wu, Super line-connectivity of consecutive-d digraphs, Discrete Mathematics 183 (1-3) (1998) 27 - 38. doi:DOI: 10.1016/S0012-365X(97)00079-4.

[36] D. Barth, M.-C. Heydemann, A new digraphs composition, Research report, LRI, Université de Paris-Sud, Orsay, France (1995).

[37] D. Barth, M.-C. Heydemann, A new digraphs composition with applications to de Bruijn and generalized de Bruijn digraphs, Discrete Applied Mathematics 77 (2) (1996) 99 - 118. doi:DOI: 10.1016/S0166218X(96)00130-8.

[38] D. Coudert, Algorithmique et optimisation de réseaux de communications optiques, Ph.D. thesis, Université de Nice Sophia-Antipolis (UNSA) (december 2001).

[39] J. Gómez, C. Padró, S. Pérennes, Large generalized cycles, Discrete Applied Mathematics 89 (1-3) (1998) 107 - 123. 